Supporting Information

\title{
Promoting Electrocatalytic Oxygen Evolution over \\ Transition-Metal Phosphide-Based Nanocomposites via Architectural and Electronic Engineering
}

Ping Li and Hua Chun Zeng*

Department of Chemical and Biomolecular Engineering, Faculty of Engineering, National University of Singapore, 10 Kent Ridge Crescent, Singapore 119260

Email: chezhc@nus.edu.sg

Table of Contents

Figures $\mathrm{S} 1$ to $\mathrm{S} 29$ Pages 2-30

Table S1

Pages 31-32

References

Pages 33-35 

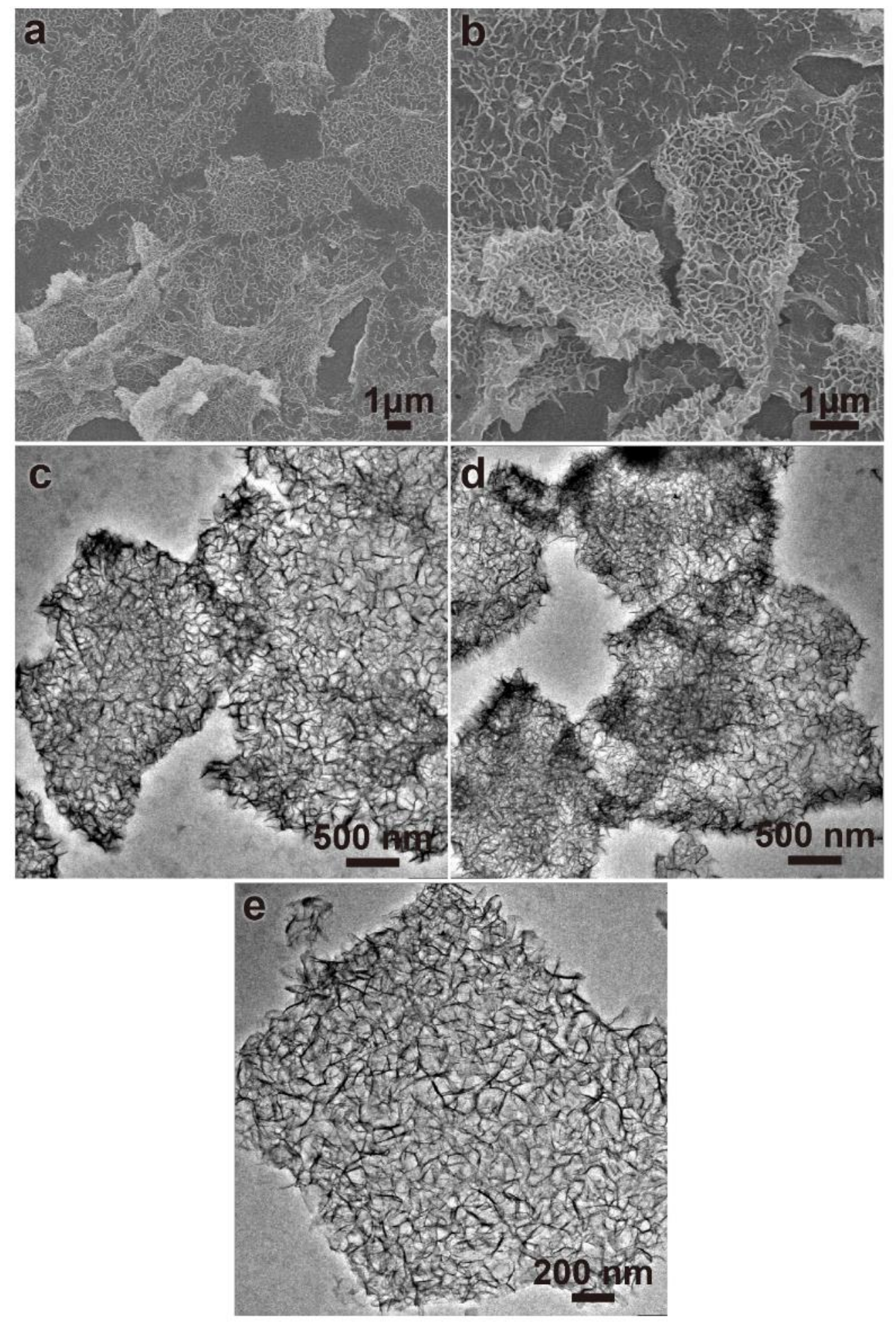

Figure S1. (a, b) SEM images and (c-e) TEM images of the rGO/CoFe-LDH sample. 


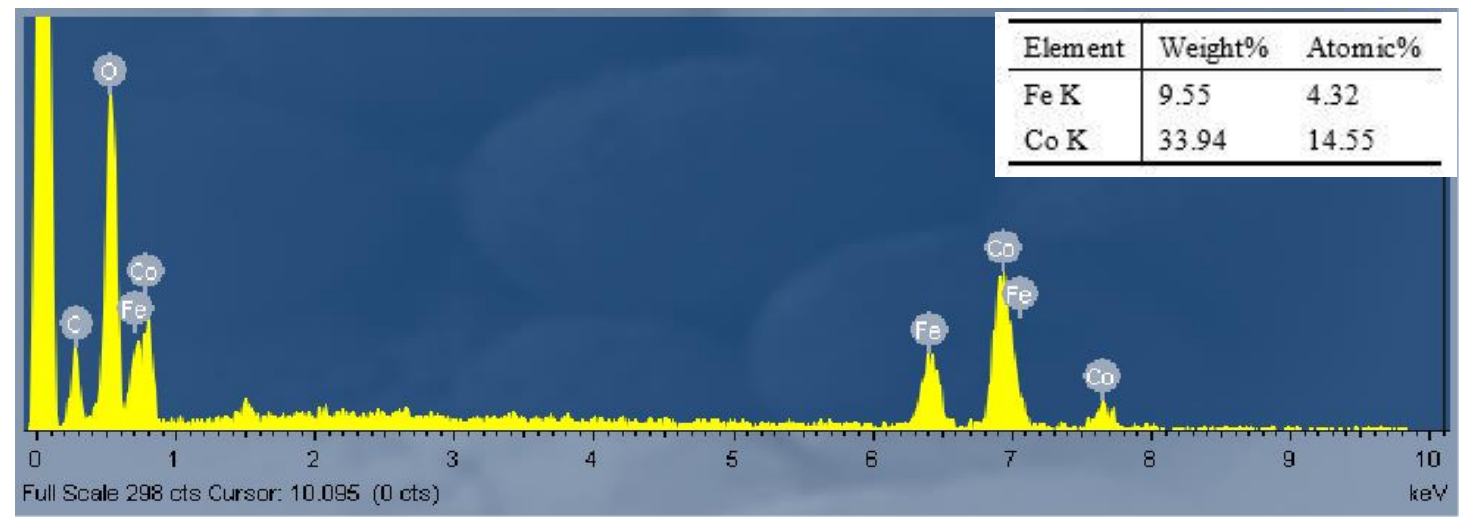

Figure S2. EDX spectrum of the $\mathrm{rGO} / \mathrm{CoFe}-\mathrm{LDH}$ sample. The atomic ratio of $\mathrm{Co}: \mathrm{Fe}$ in this sample is about $3: 1$. 


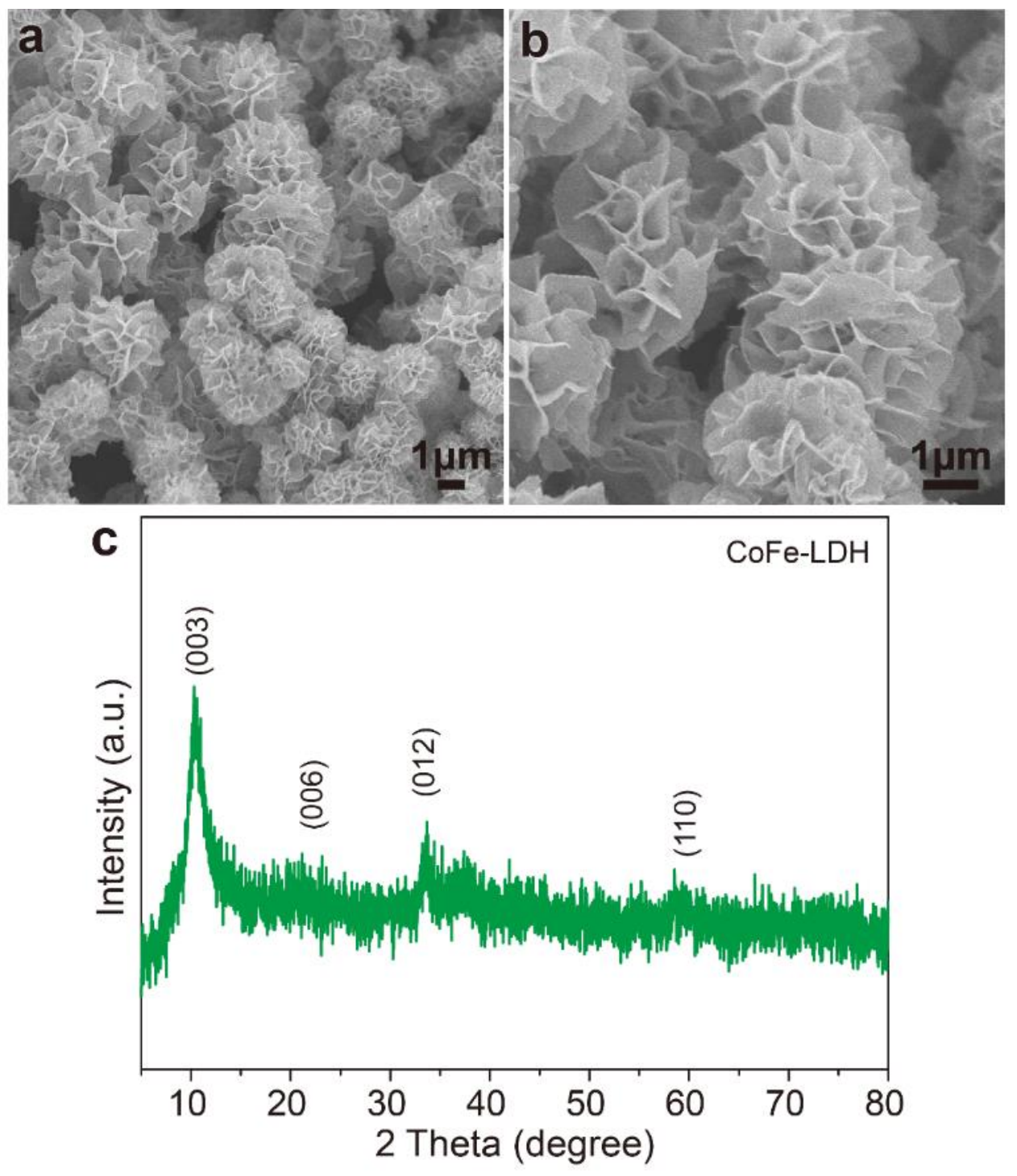

Figure S3. Characterization of the free-standing CoFe-LDH sample: (a, b) SEM images, and (c) XRD pattern. 


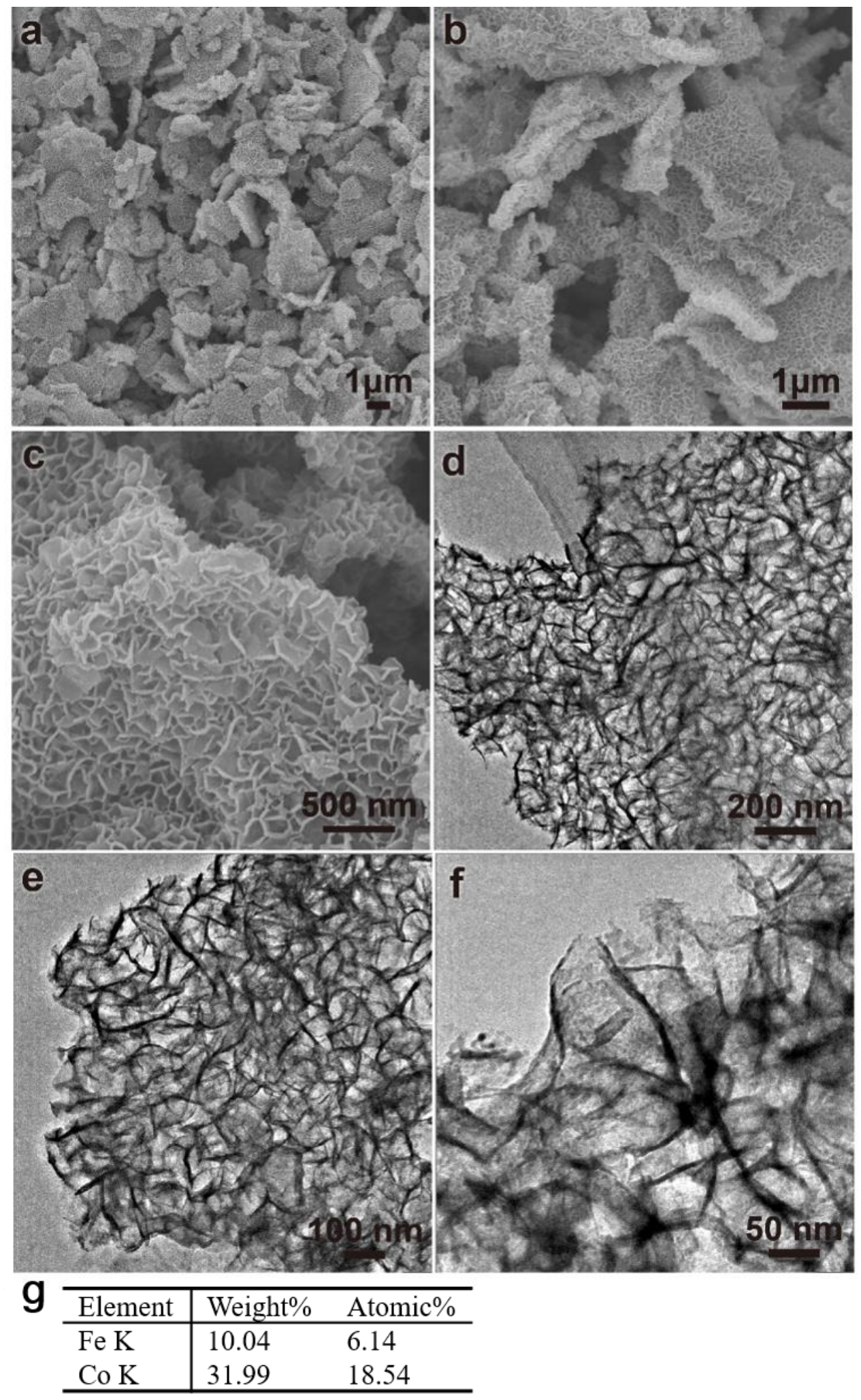

Figure S4. (a-c) SEM images and (d-f) TEM images of the rGO/CoFe-P sample. (g)

The metal atomic ratio in the $\mathrm{rGO} / \mathrm{CoFe}-\mathrm{P}$ sample from the EDX analysis. 


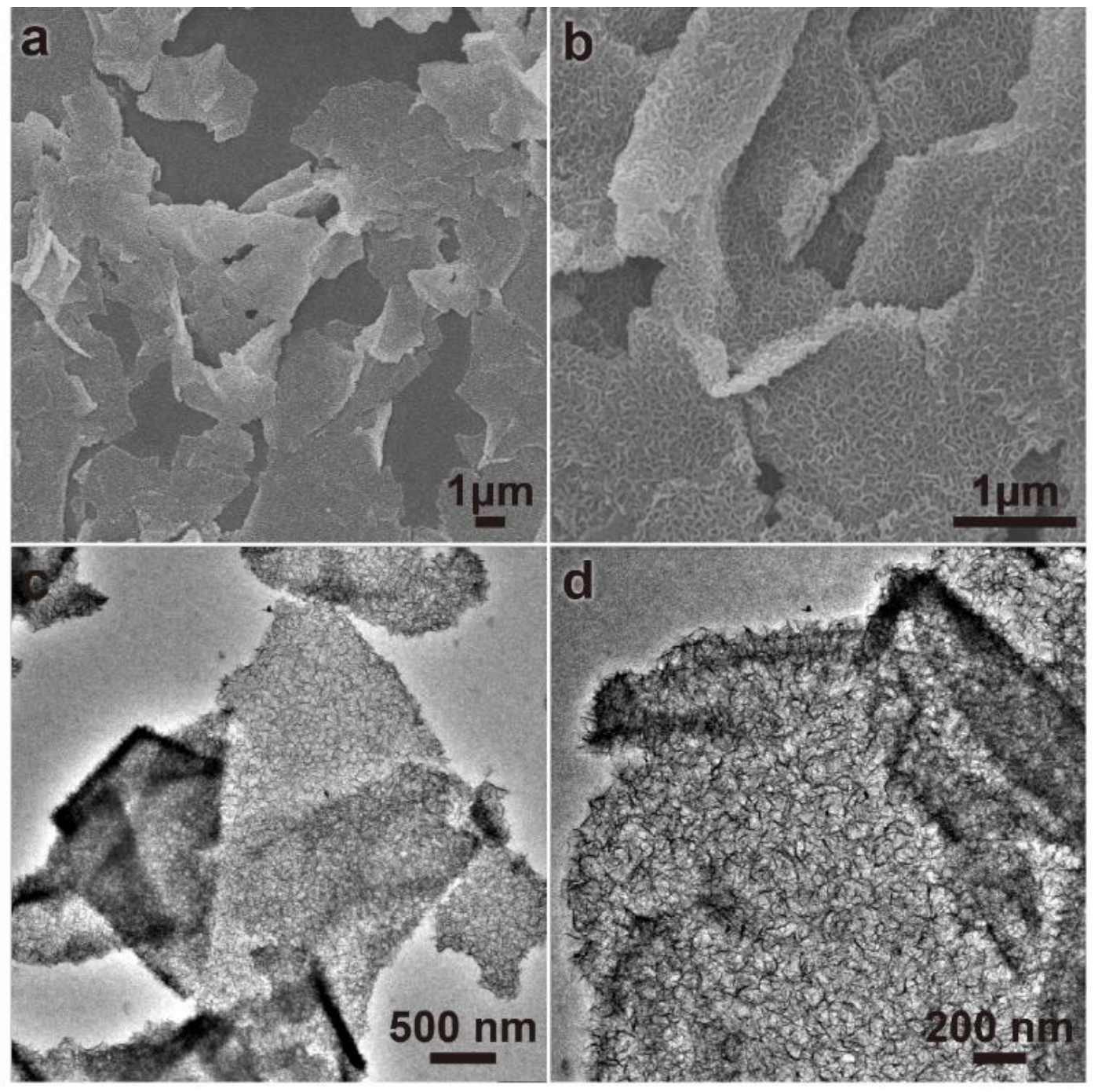

Figure S5. (a, b) SEM images and (c, d) TEM images of the rGO/NiFe-LDH sample. 

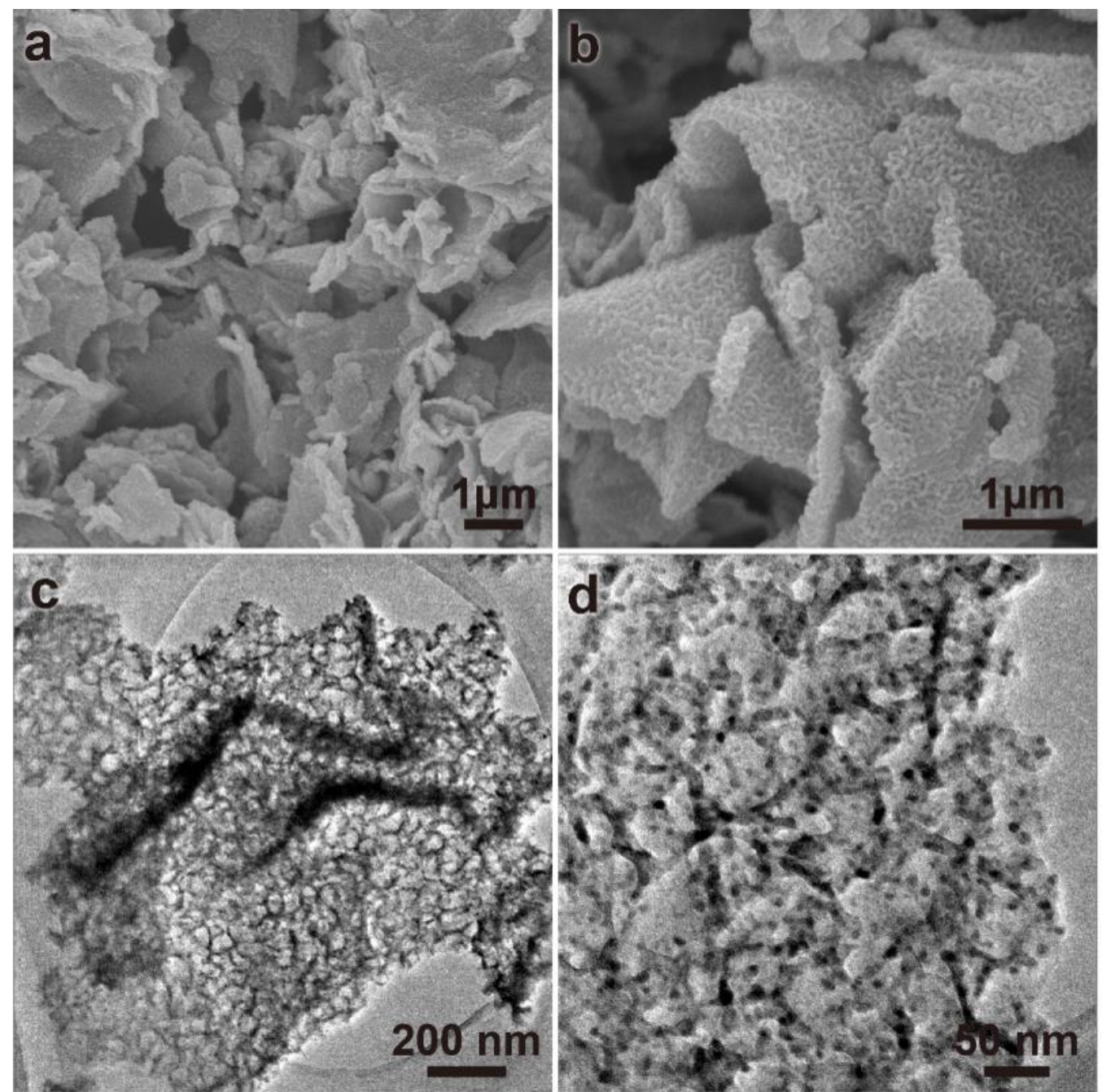

\begin{tabular}{l|ll}
\hline Element & Weight $\%$ & Atomic $\%$ \\
\hline Fe K & 7.75 & 4.85 \\
Ni K & 23.52 & 13.97 \\
\hline
\end{tabular}

Figure S6. (a, b) SEM images and (c, d) TEM images of the rGO/NiFe-P sample. (e)

The metal atomic ratio in the $\mathrm{rGO} / \mathrm{NiFe}-\mathrm{P}$ sample from the EDX analysis. 

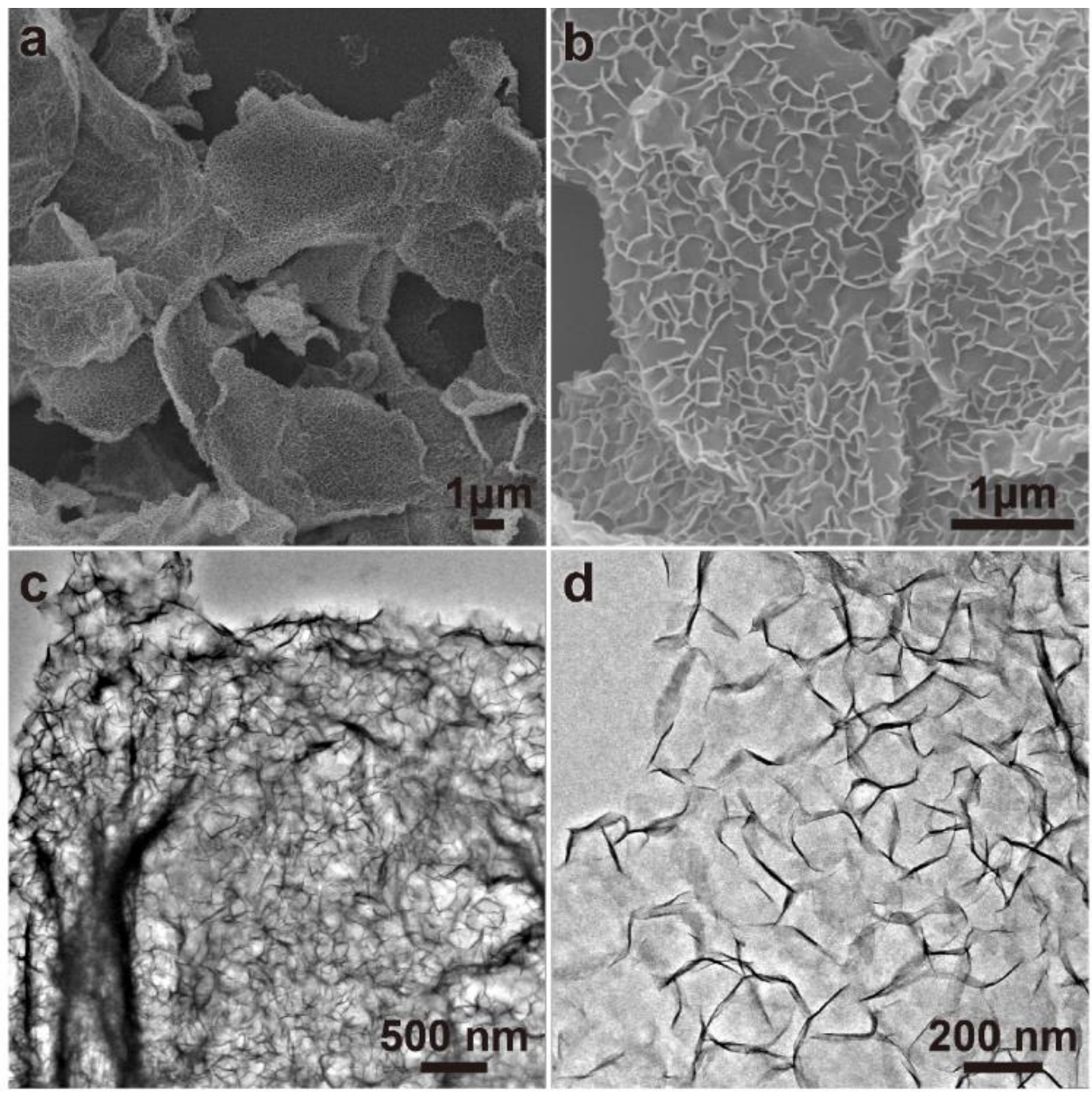

Figure S7. (a, b) SEM mages and (c, d) TEM images of the rGO/CoNi-LDH sample. 

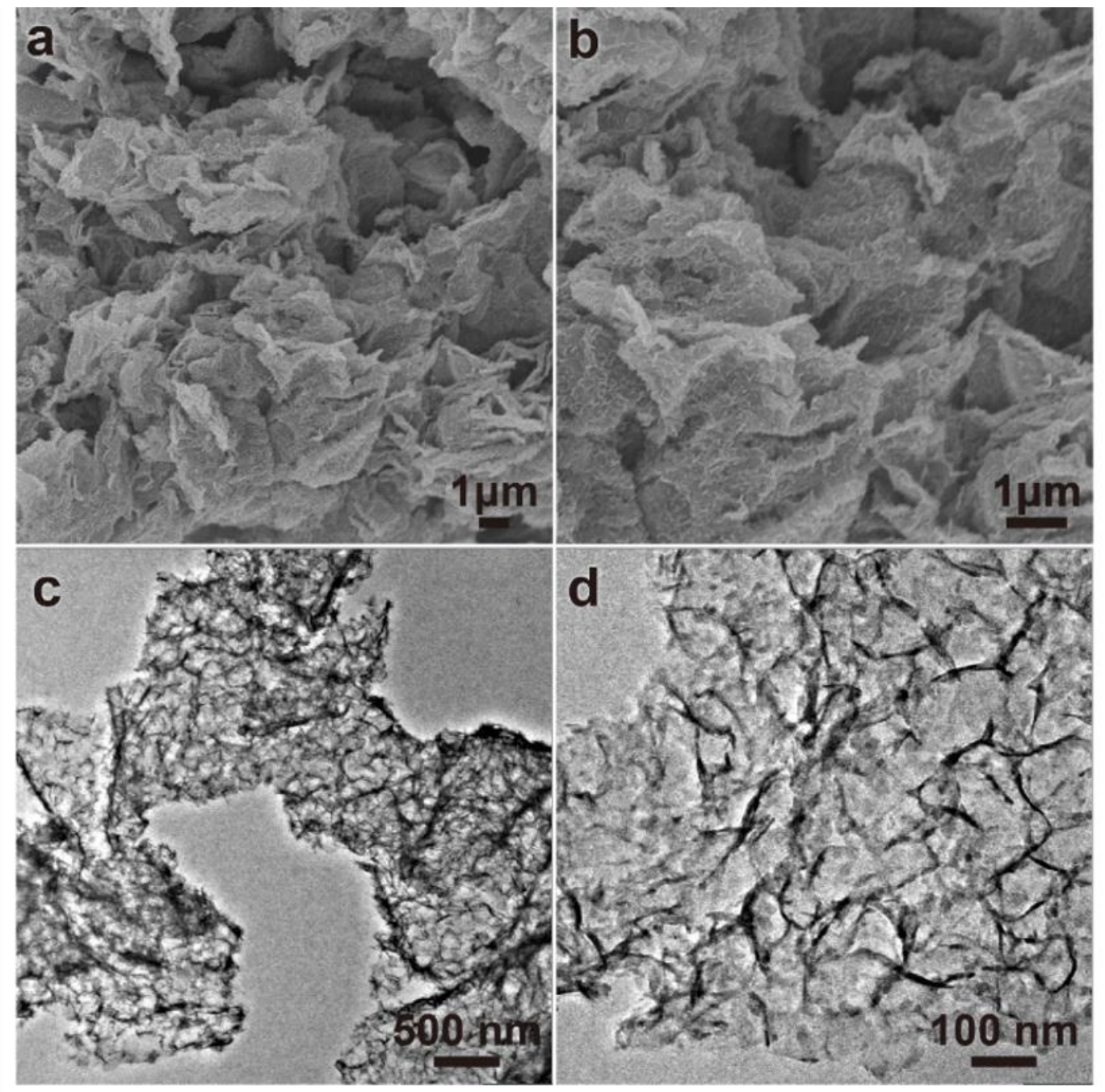

\begin{tabular}{l|ll}
\hline Element & Weight $\%$ & Atomic\% \\
\hline Co K & 13.85 & 11.61 \\
Ni K & 15.16 & 12.75 \\
\hline
\end{tabular}

Figure S8. (a, b) SEM images and (c, d) TEM images of the rGO/CoNi-P sample. (e)

The metal atomic ratio in the $\mathrm{rGO} / \mathrm{CoNi}-\mathrm{P}$ sample from the EDX analysis. 

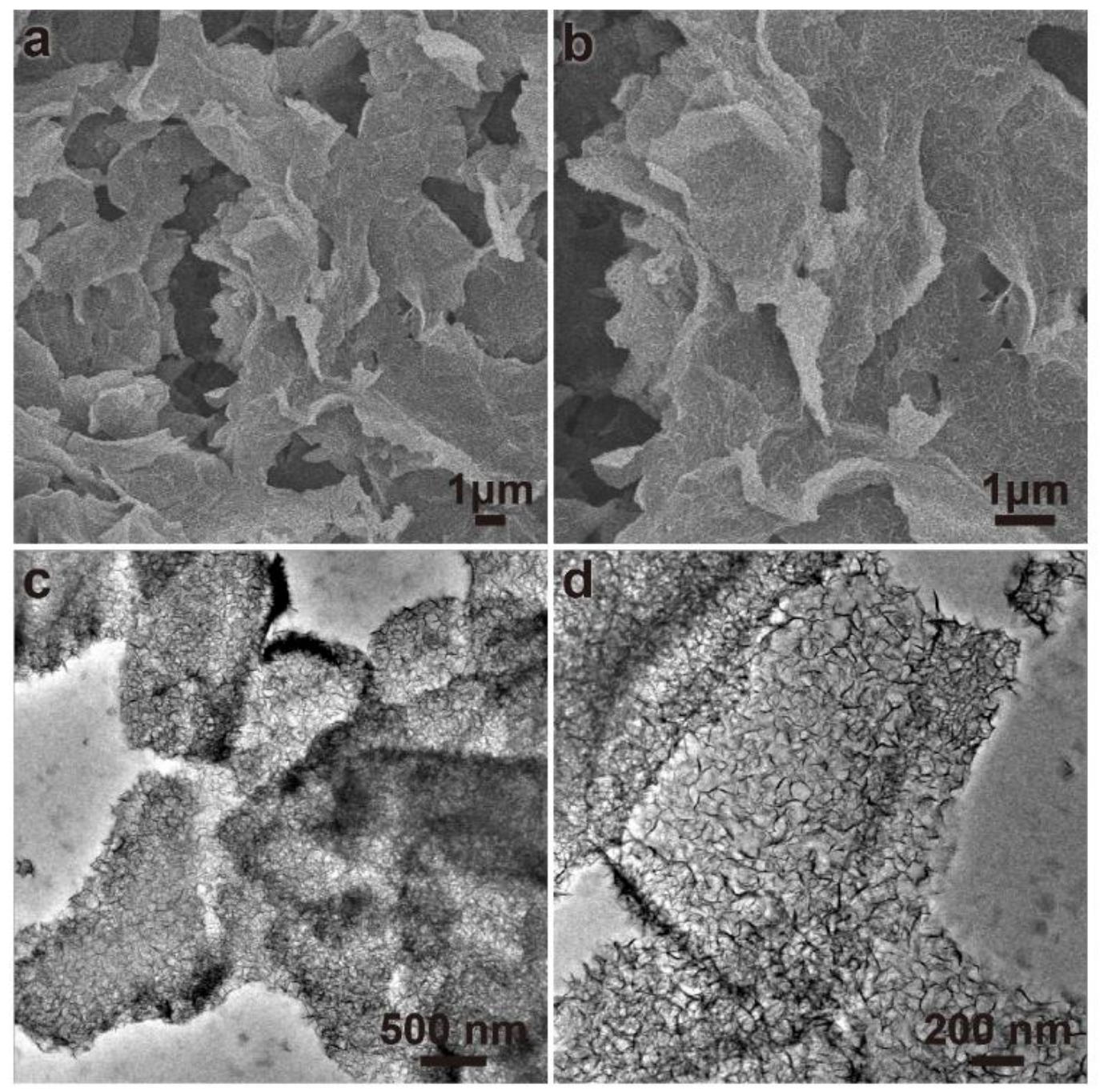

Figure S9. (a, b) SEM images and (c, d) TEM images of the rGO/CoNiFe-LDH sample. 


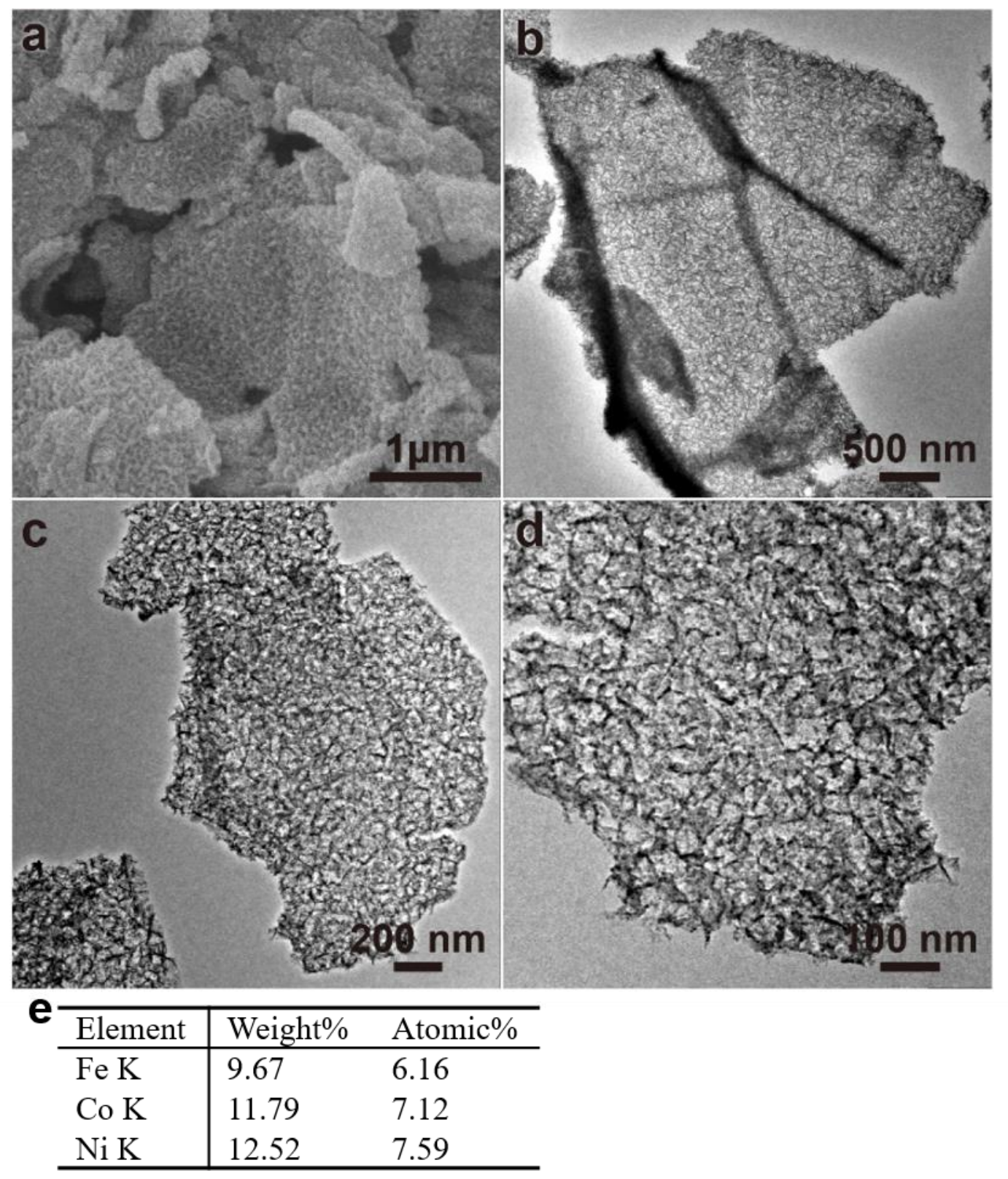

Figure S10. (a, b) SEM images and (c, d) TEM images of the rGO/CoNiFe-P sample.

(e) The metal atomic ratio in the $\mathrm{rGO} / \mathrm{CoNiFe}-\mathrm{P}$ sample from the EDX analysis. 

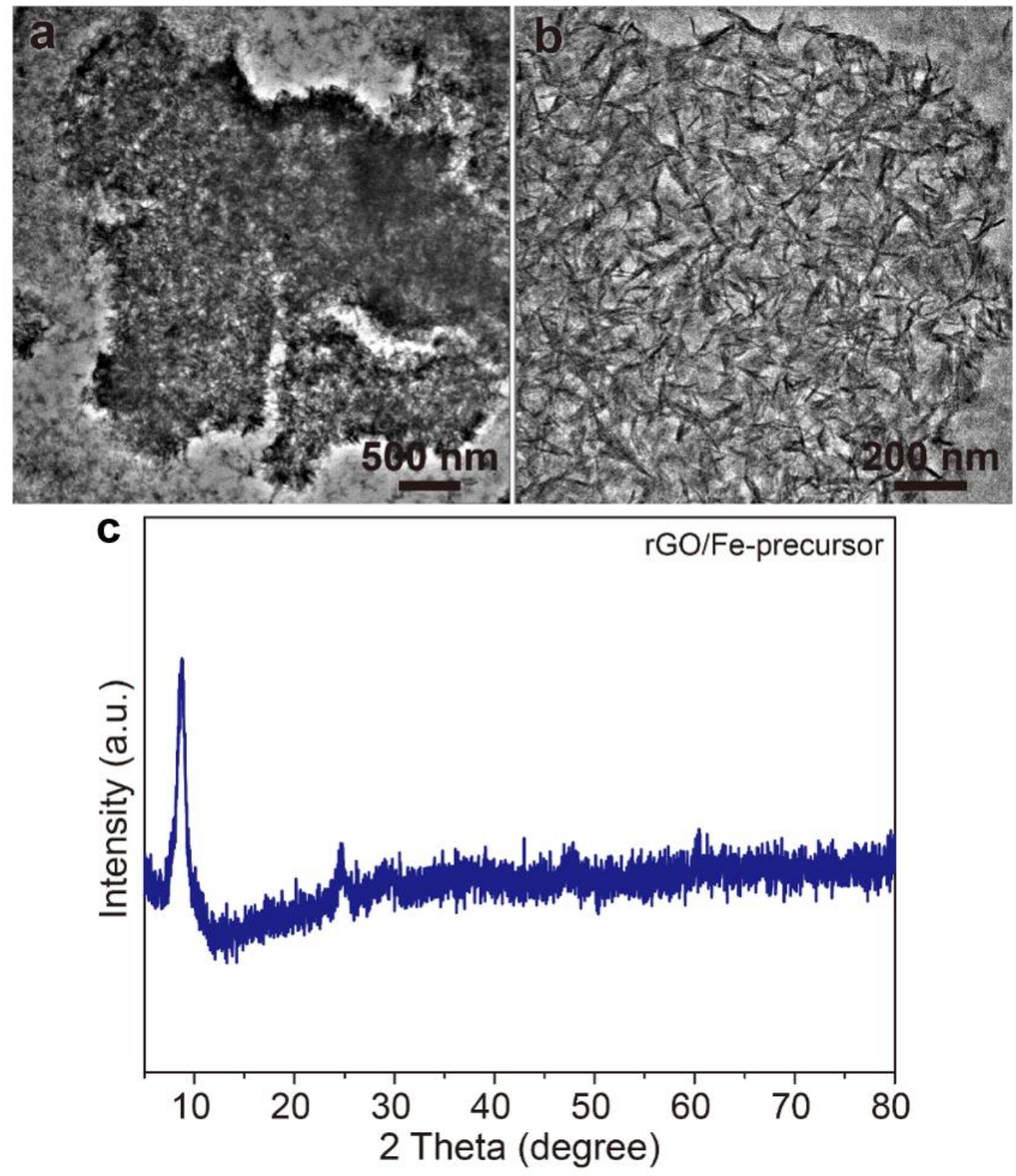

Figure S11. Characterization of the $\mathrm{rGO} / \mathrm{Fe}$-precursor: $(\mathrm{a}, \mathrm{b}) \mathrm{TEM}$ images, and (c) XRD pattern. 

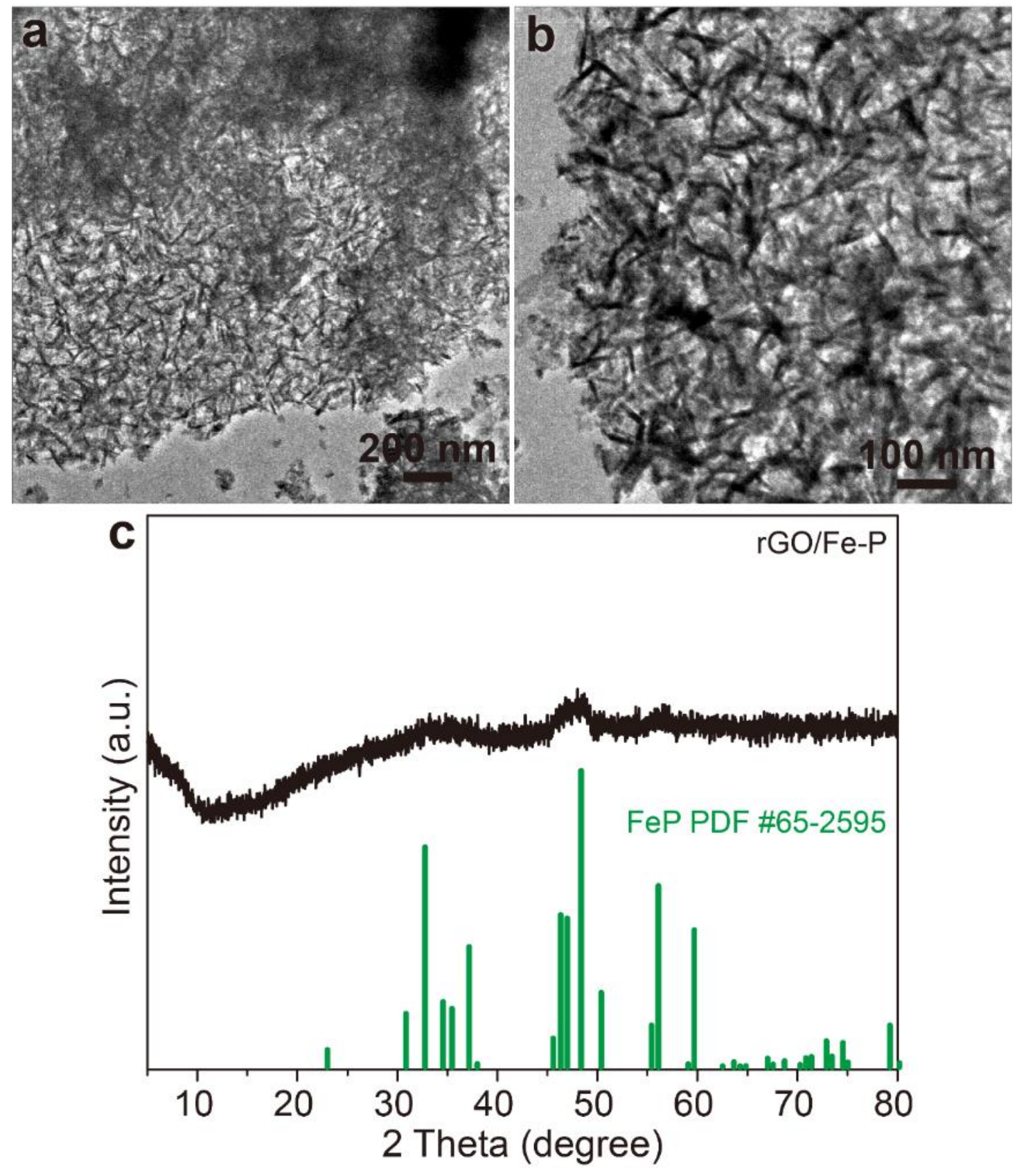

Figure S12. Characterization of the rGO/Fe-P sample: (a, b) TEM images, and (c) XRD pattern and reference XRD data. 

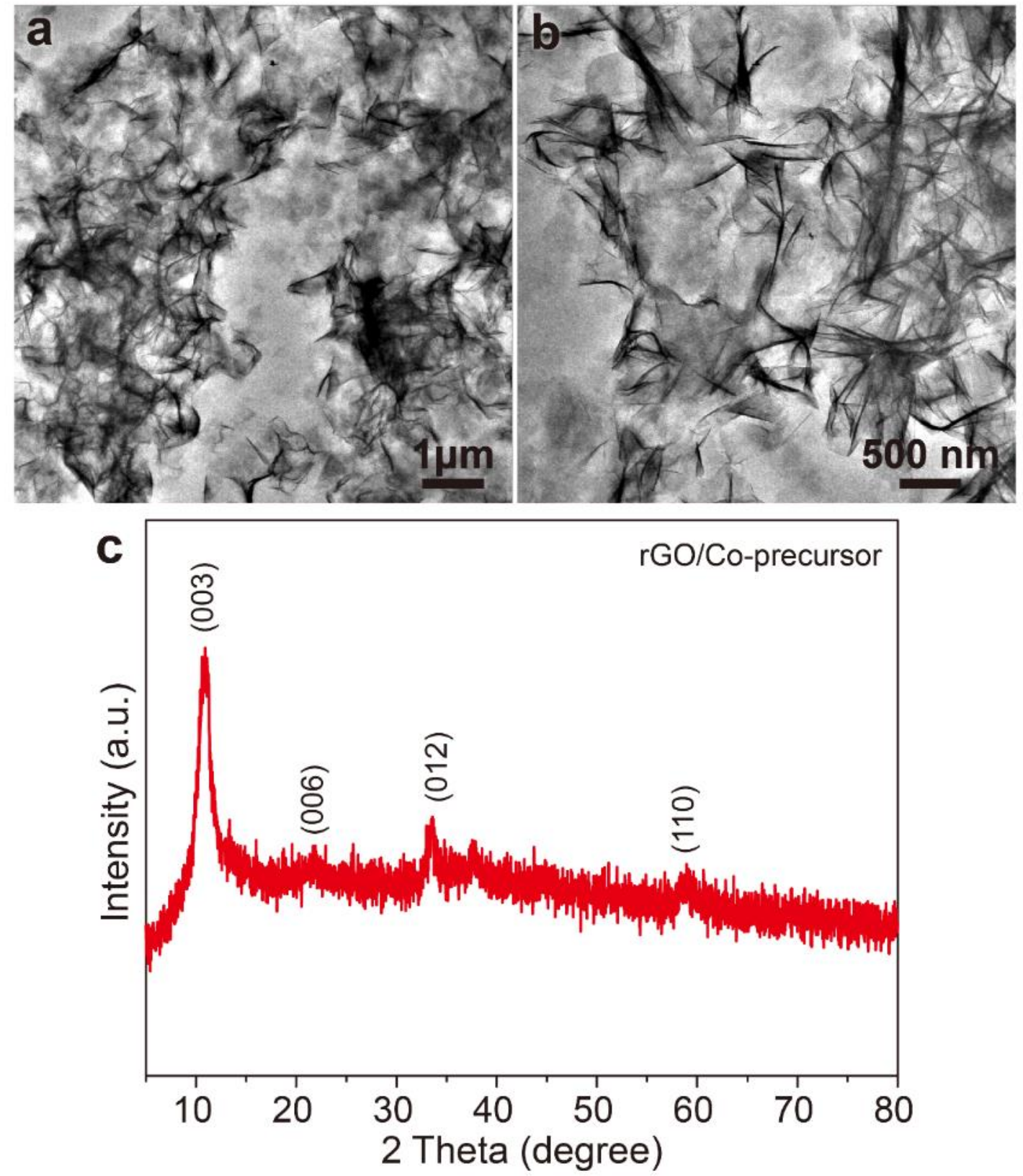

Figure S13. Characterization of the rGO/Co-precursor: (a, b) TEM images, and (c) XRD pattern. 

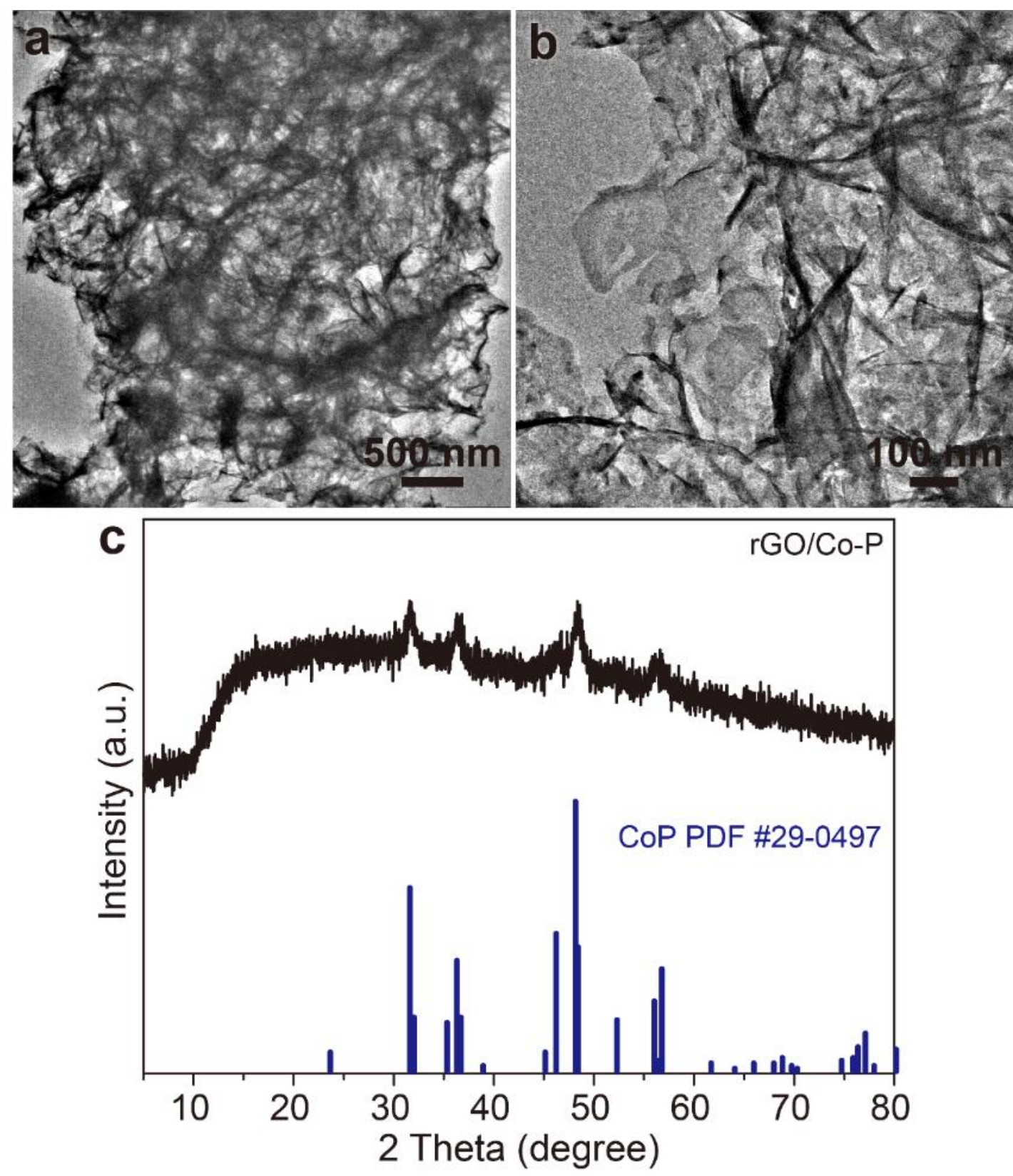

Figure S14. Characterization of the rGO/Co-P sample: (a, b) TEM images, and (c) XRD pattern and reference XRD data. 

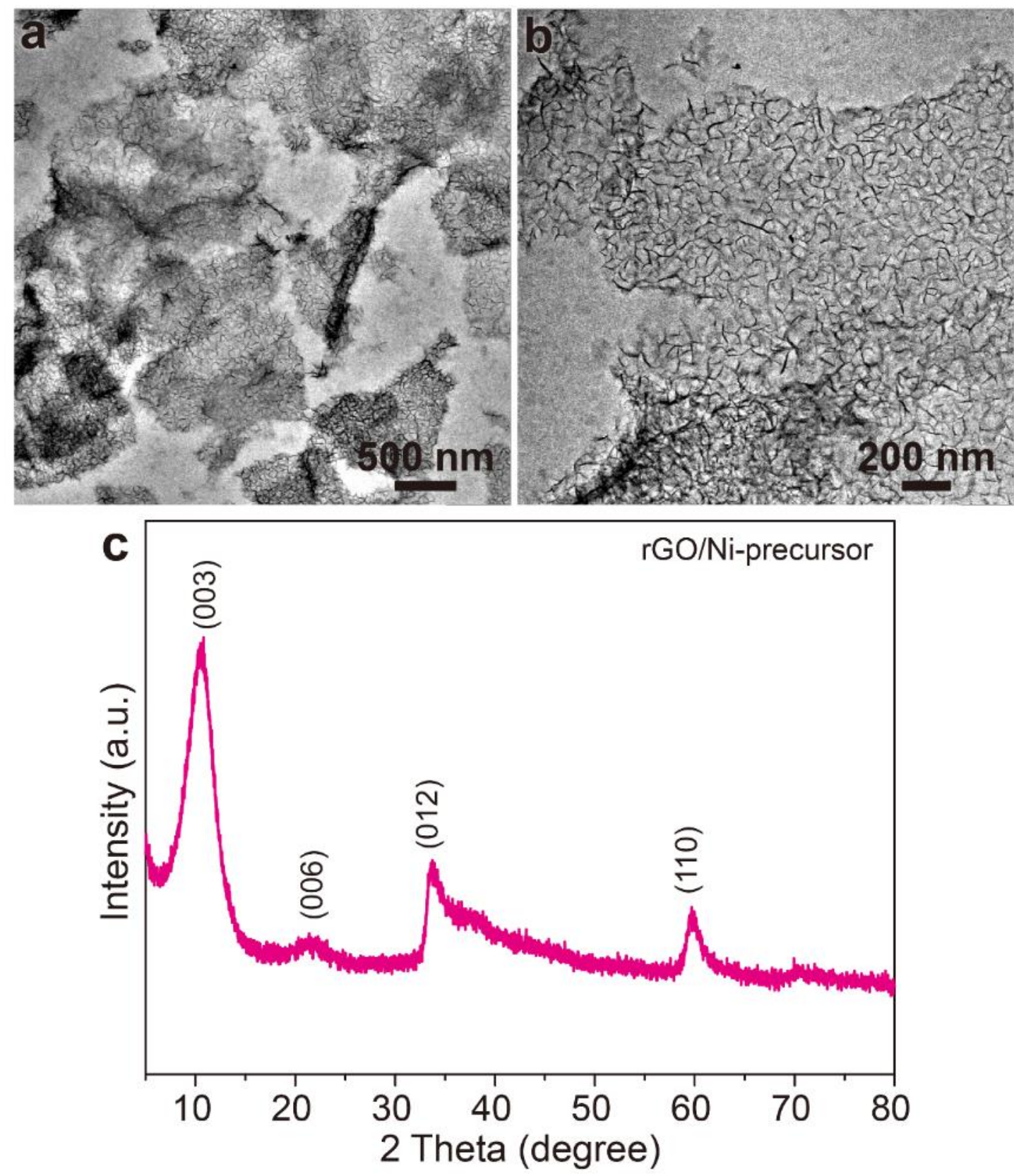

Figure S15. Characterization of the rGO/Ni-precursor: $(a, b)$ TEM images, and (c) XRD pattern. 

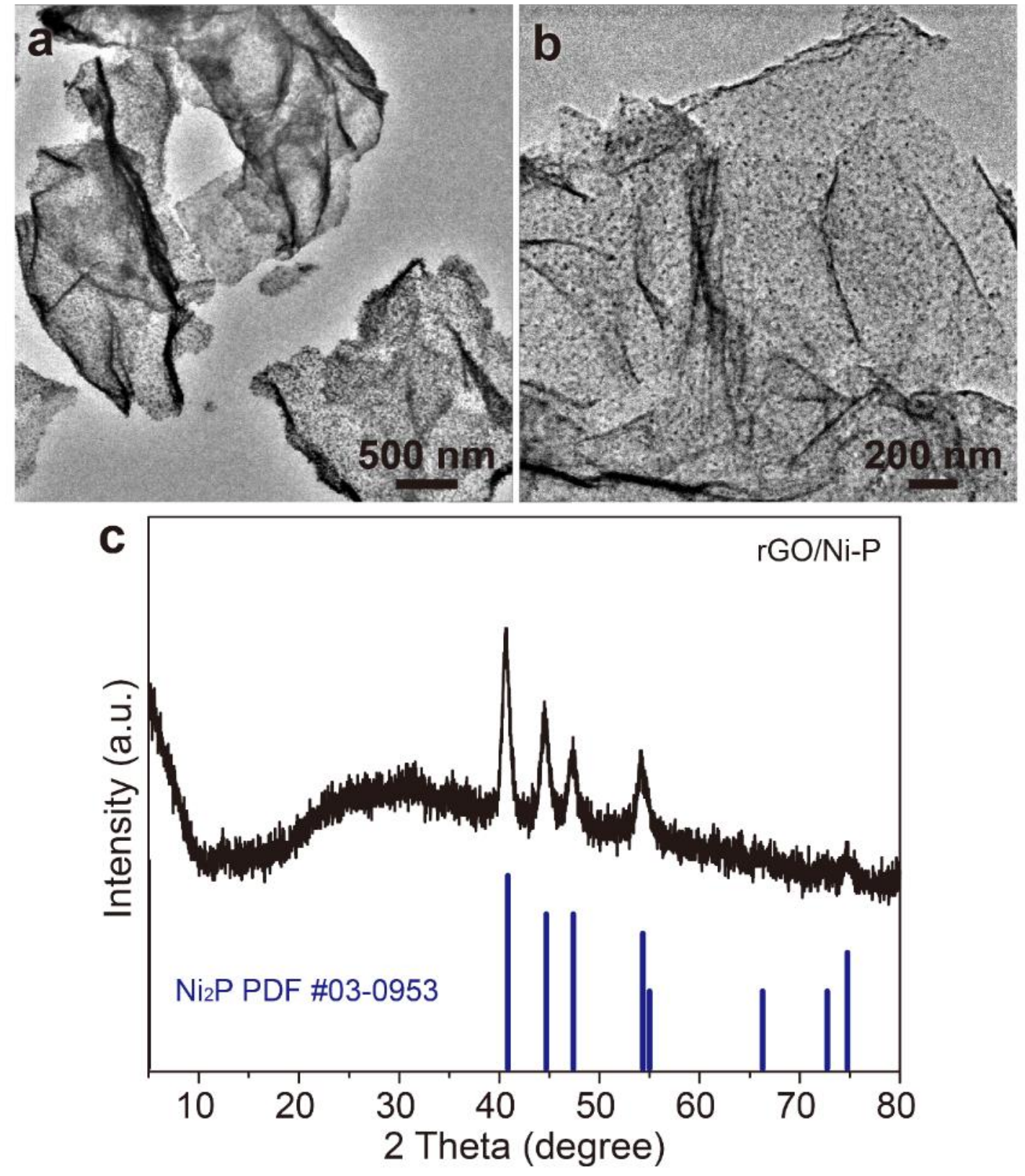

Figure S16. Characterization of the rGO/Ni-P sample: (a, b) TEM images, and (c) XRD pattern and reference XRD data. 

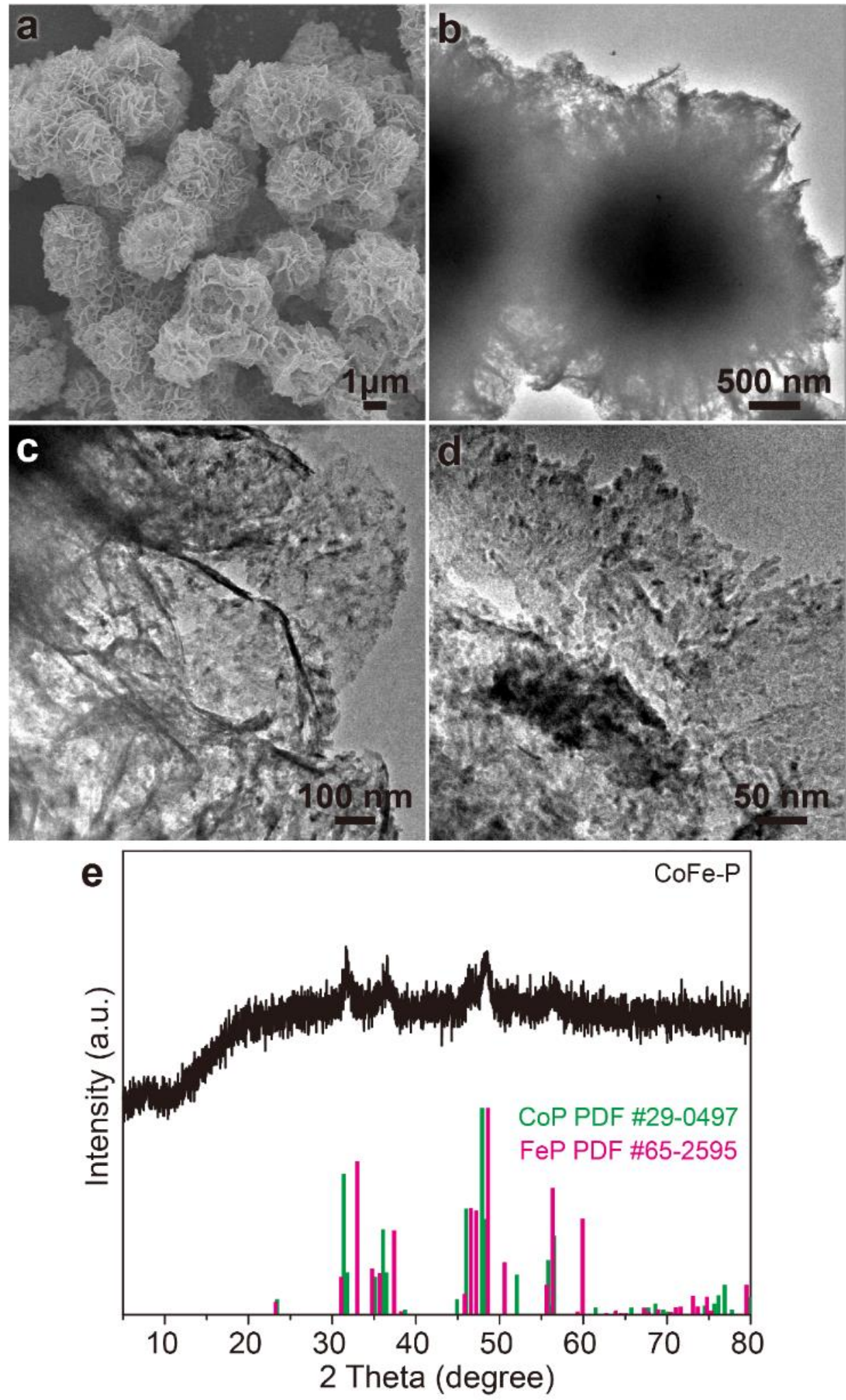

Figure S17. Characterization of the free-standing CoFe-P sample: (a) SEM image, (bd) TEM images, and (e) XRD pattern and reference XRD data. 

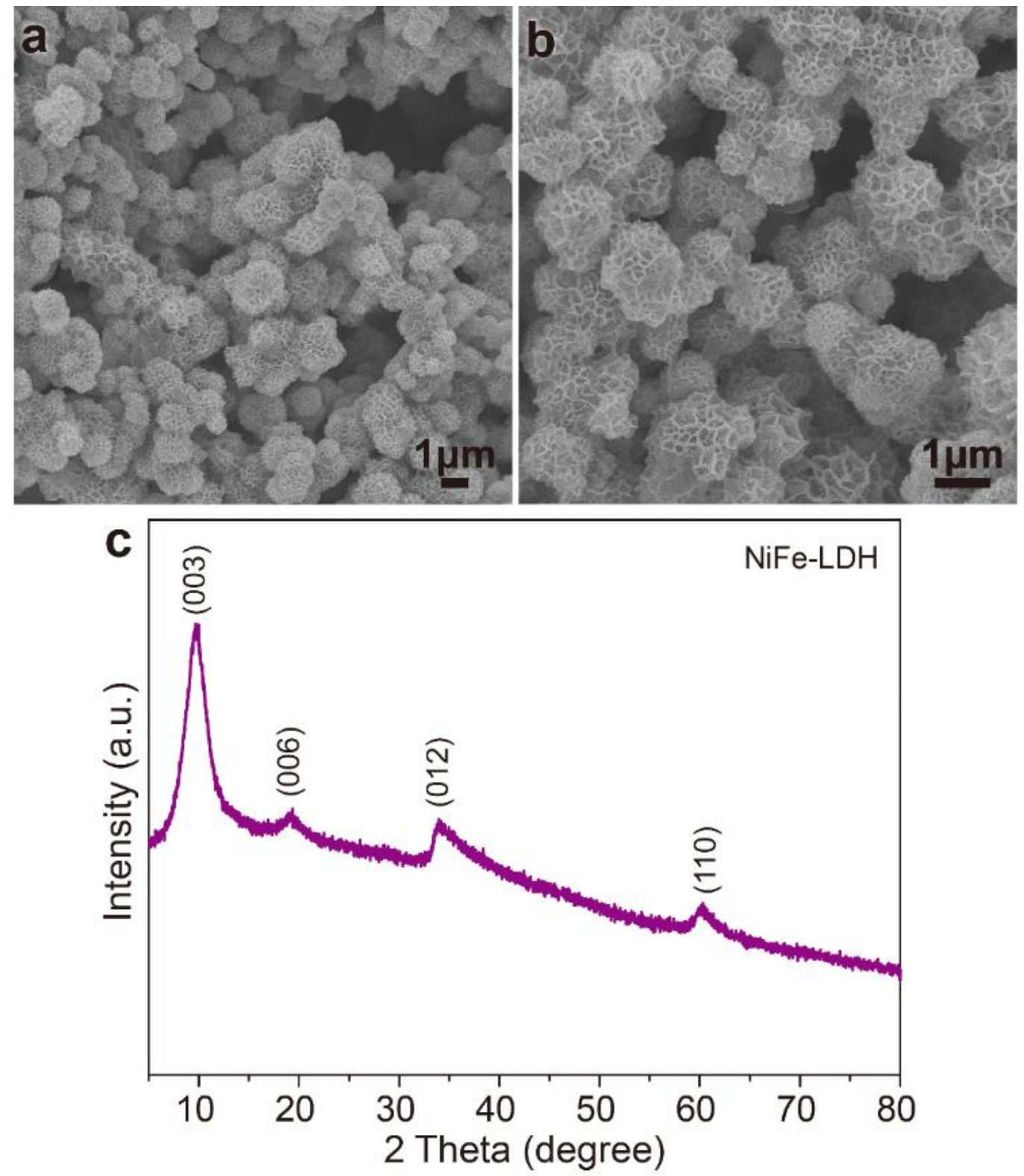

Figure S18. Characterization of the free-standing NiFe-LDH sample: (a, b) SEM images, and (c) XRD pattern. 


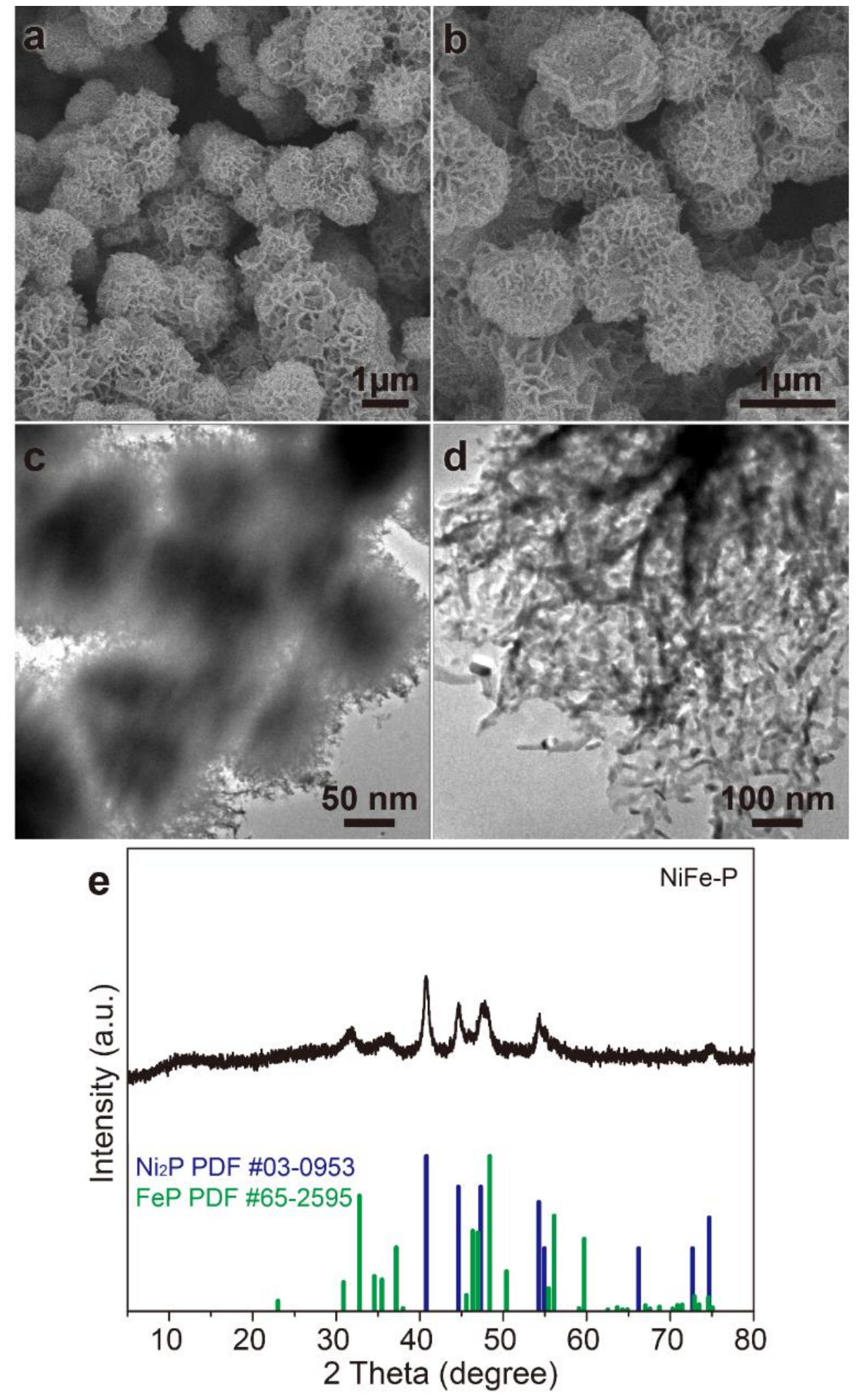

Figure S19. Characterization of the free-standing NiFe-P sample: (a, b) SEM images, (c, d) TEM images, and (e) XRD pattern and reference XRD data. 

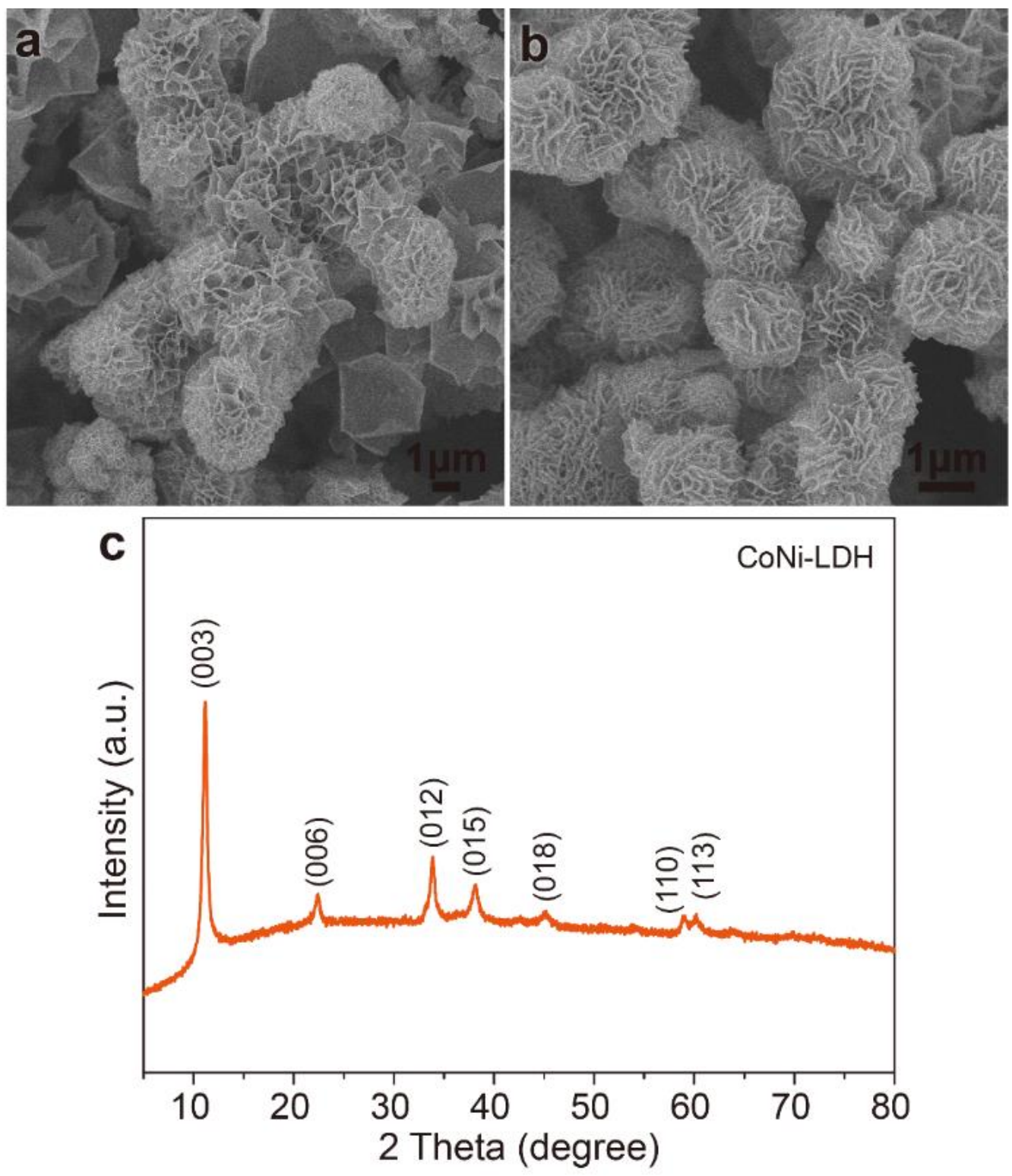

Figure S20. Characterization of the free-standing CoNi-LDH sample: (a, b) SEM images, and (c) XRD pattern. 


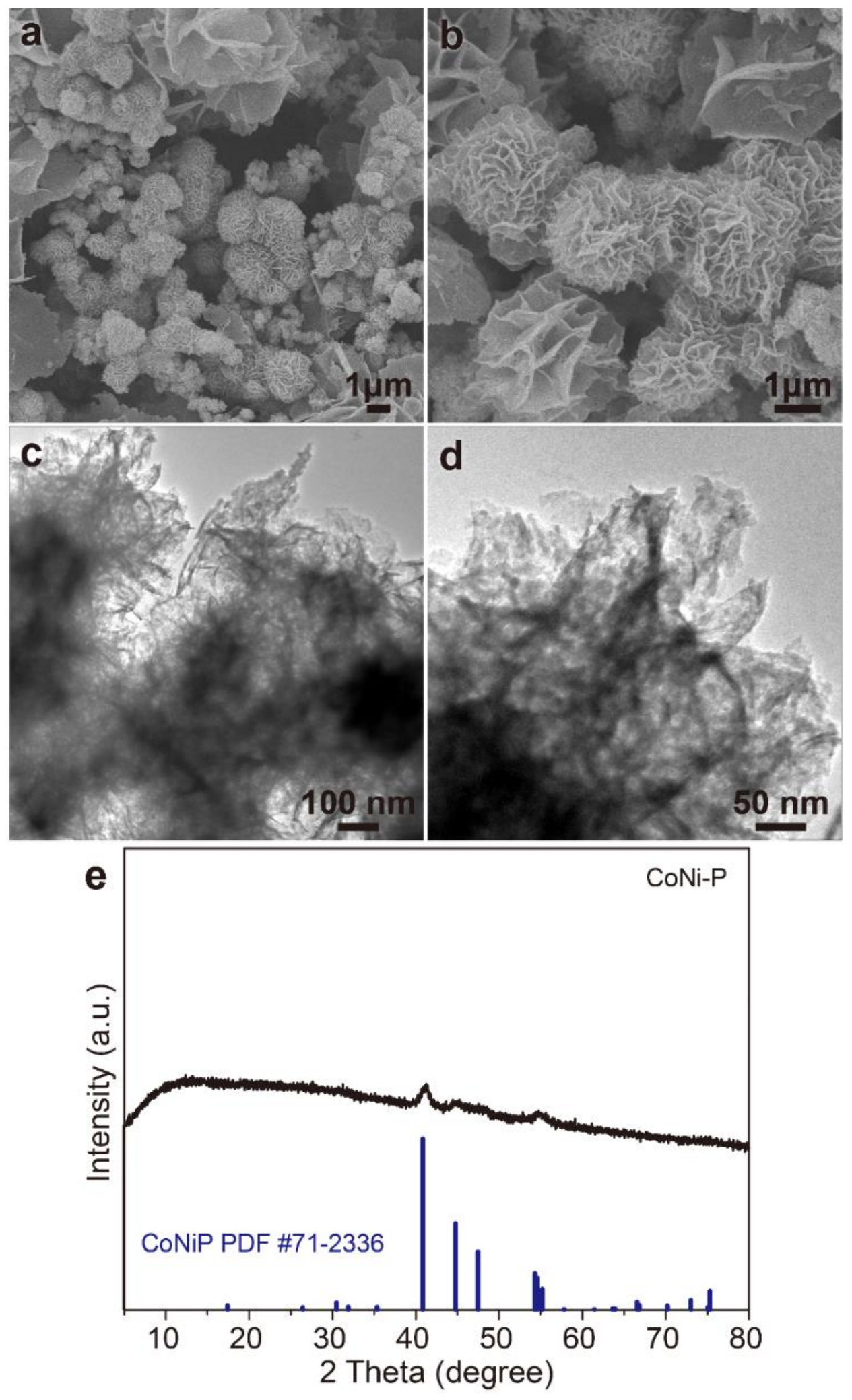

Figure S21. Characterization of the free-standing CoNi-P sample: (a, b) SEM images, (c, d) TEM images, and (e) XRD pattern and reference XRD data. 

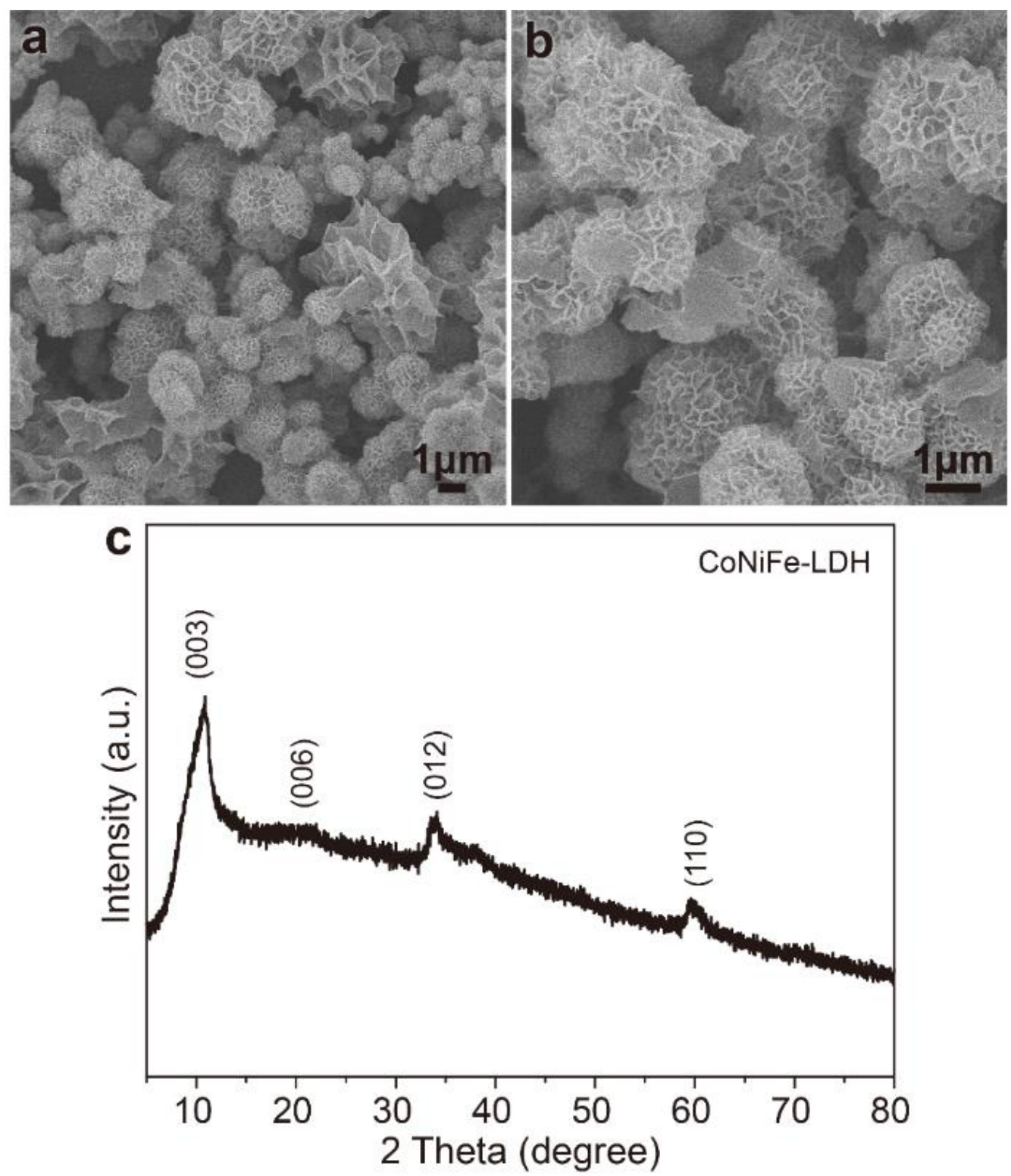

Figure S22. Characterization of the free-standing CoNiFe-LDH sample: (a, b) SEM images, and (c) XRD pattern. 

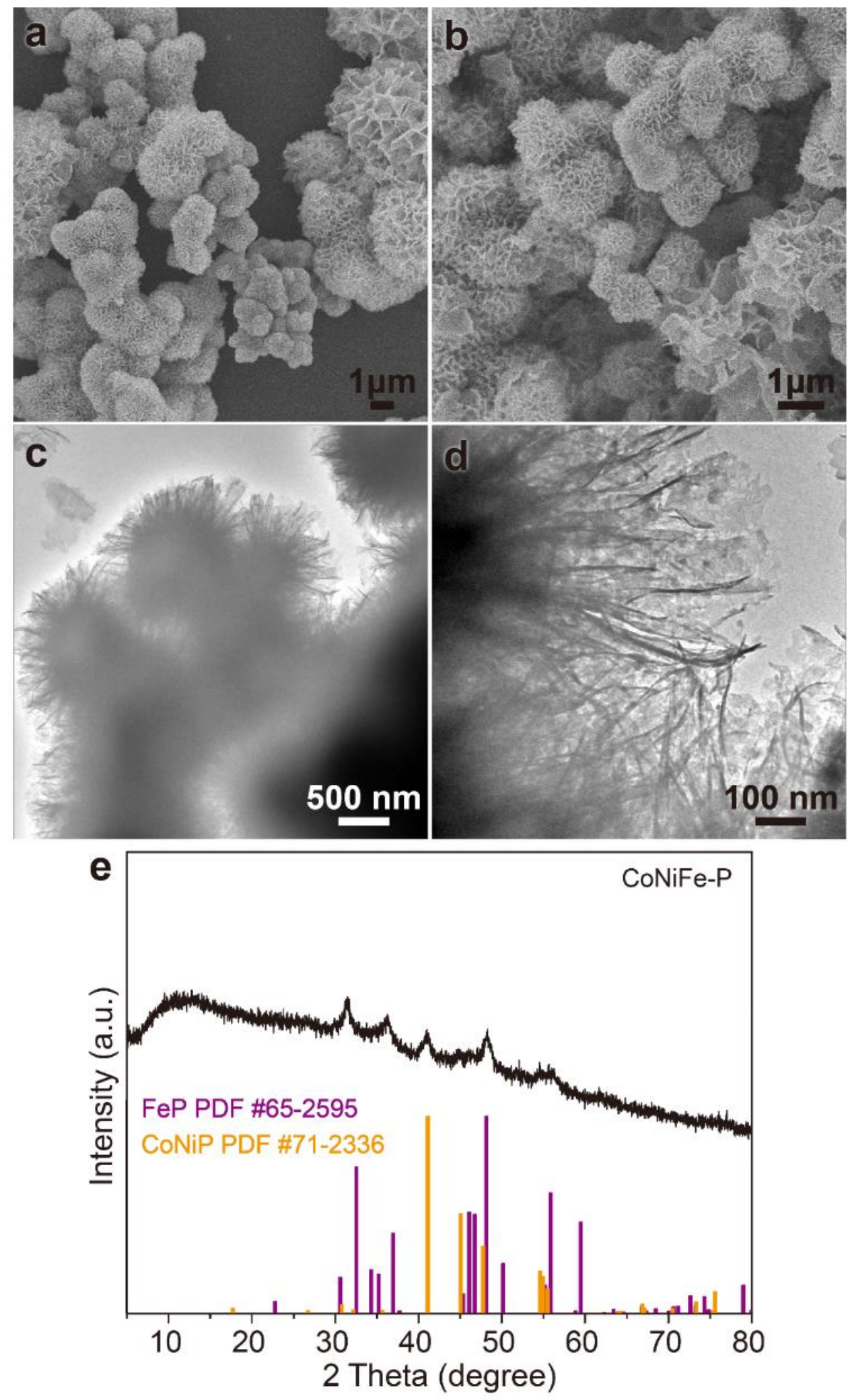

Figure S23. Characterization of the free-standing CoNiFe-P sample: (a, b) SEM images, (c, d) TEM images, and (e) XRD pattern and reference XRD data. 


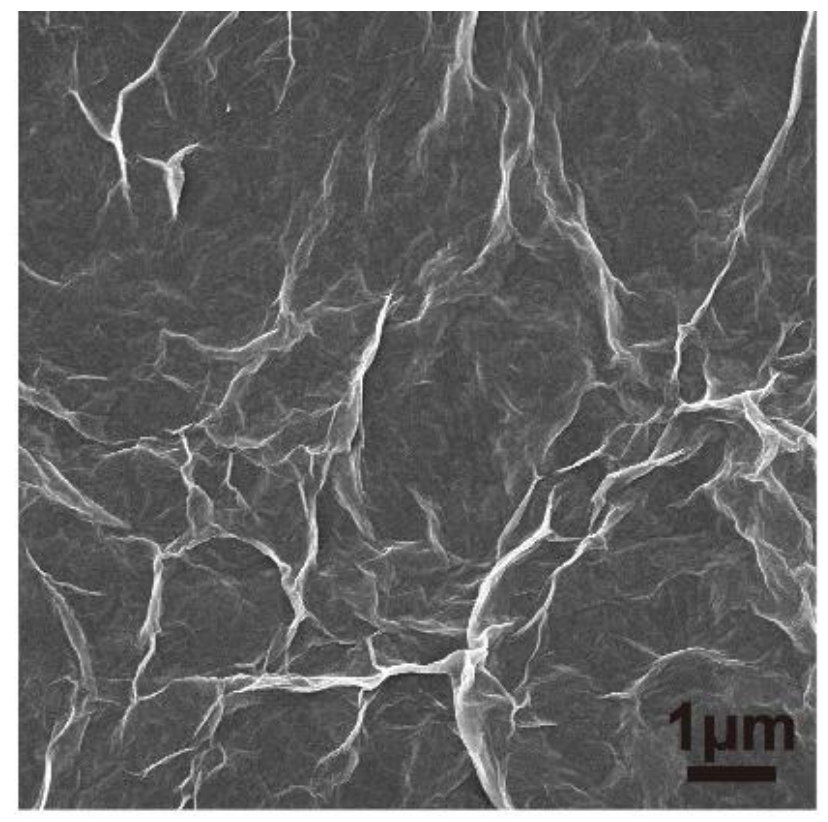

Figure S24. The SEM image of the pure rGO sample. 

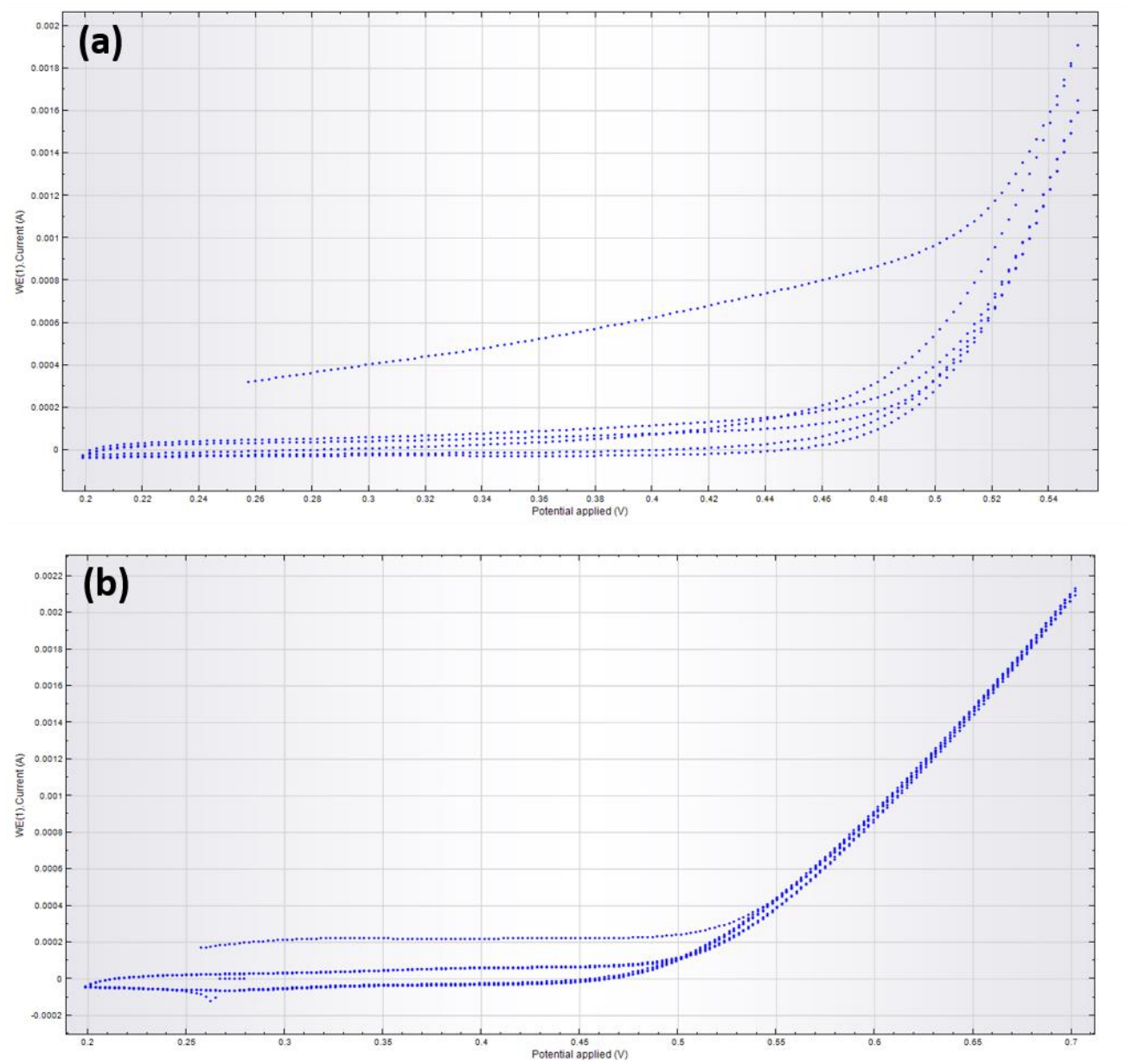

Figure S25. The initial CV curves (without iR compensation) of (a) the $\mathrm{rGO} / \mathrm{CoFe}-\mathrm{P}$ and (b) the rGO/CoFe-LDH before collecting the polarization curves. 


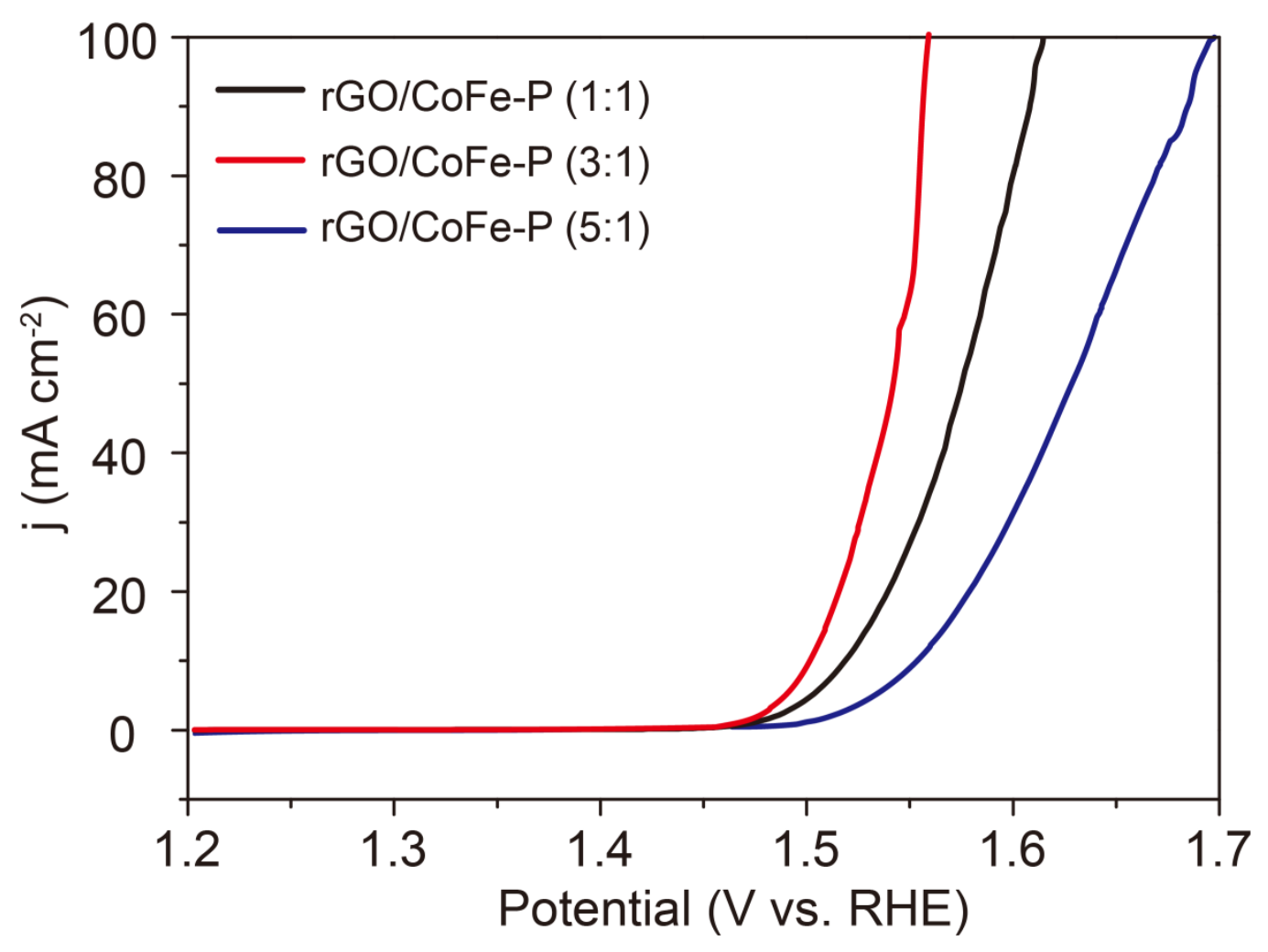

Figure S26. Polarization curves of the $\mathrm{rGO} / \mathrm{CoFe}-\mathrm{P}$ samples with different $\mathrm{Co}: \mathrm{Fe}$ atomic ratios (i.e., $\mathrm{Co}: \mathrm{Fe}=1: 1,3: 1$, and 5:1). 

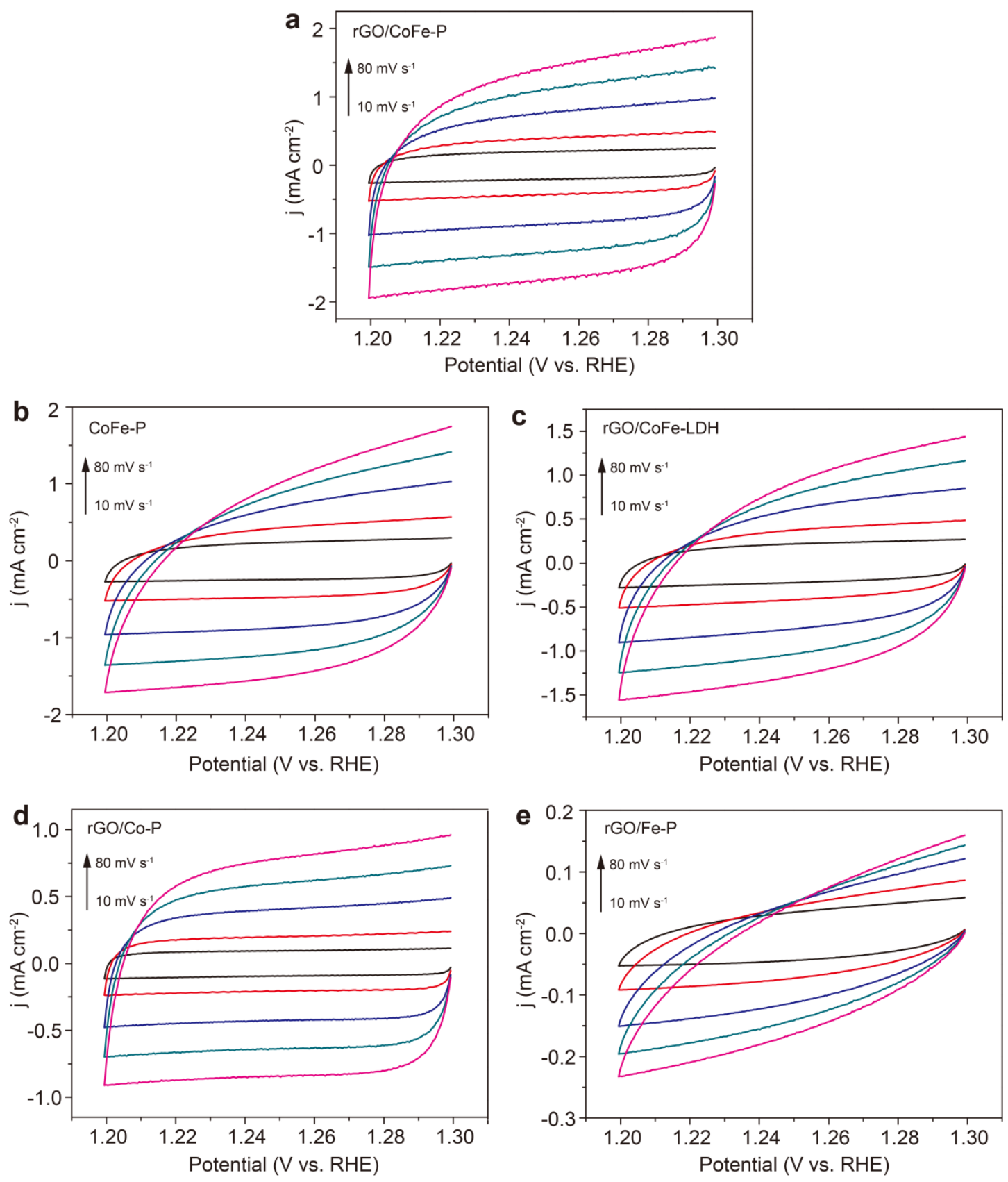

Figure S27. Cyclic voltammetry curves of 5 studied samples: (a) rGO/CoFe-P, (b) freestanding CoFe-P, (c) rGO/CoFe-LDH, (d) rGO/Co-P, and (e) rGO/Fe-P in a nonFaradaic region (1.20 to $1.30 \mathrm{~V}$ vs RHE) with different scan rates $(10,20,40,60$ and $80 \mathrm{mV} \mathrm{s}^{-1}$ ) in $1.0 \mathrm{M} \mathrm{KOH}$. 


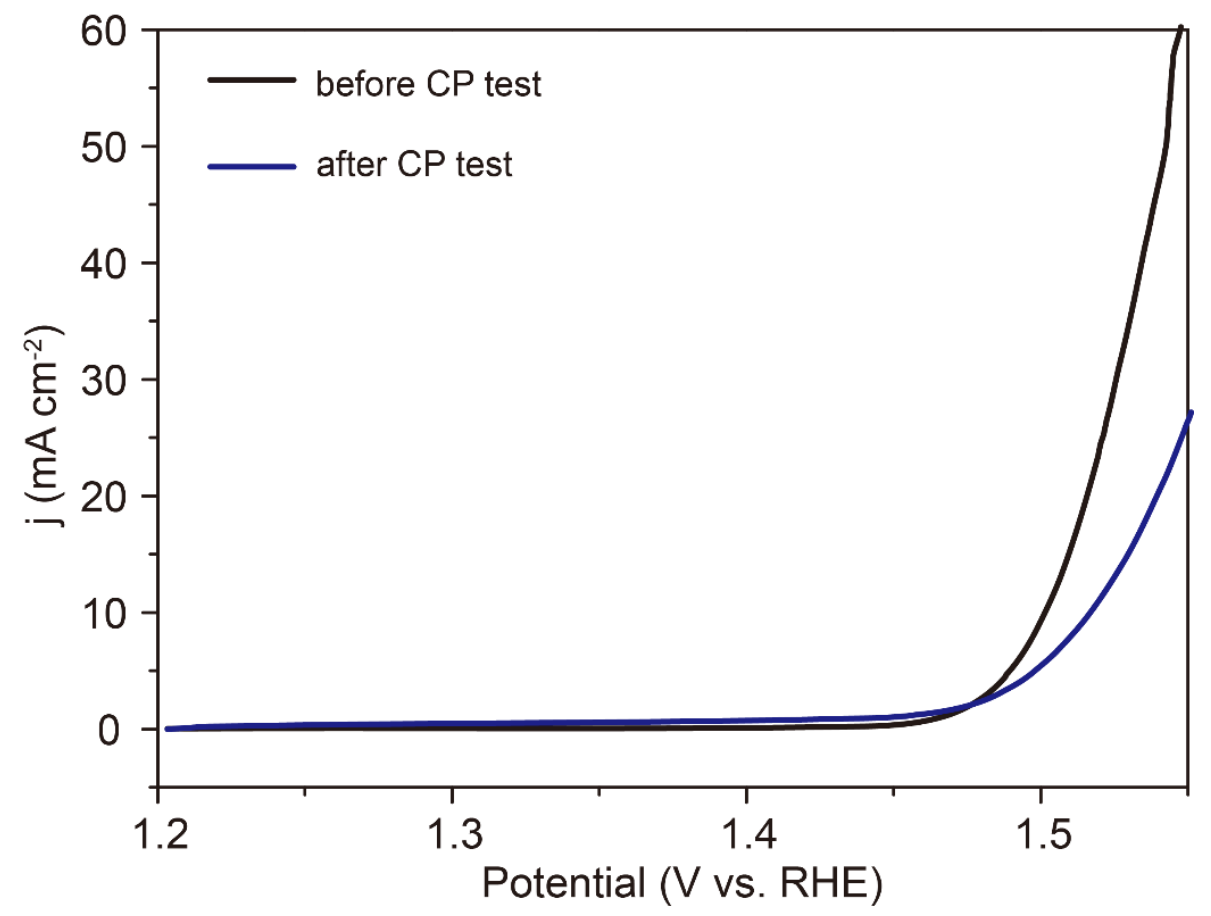

Figure S28. Polarization curves of the $\mathrm{rGO} / \mathrm{CoFe}-\mathrm{P}$ before and after $\mathrm{CP}$ stability test. 

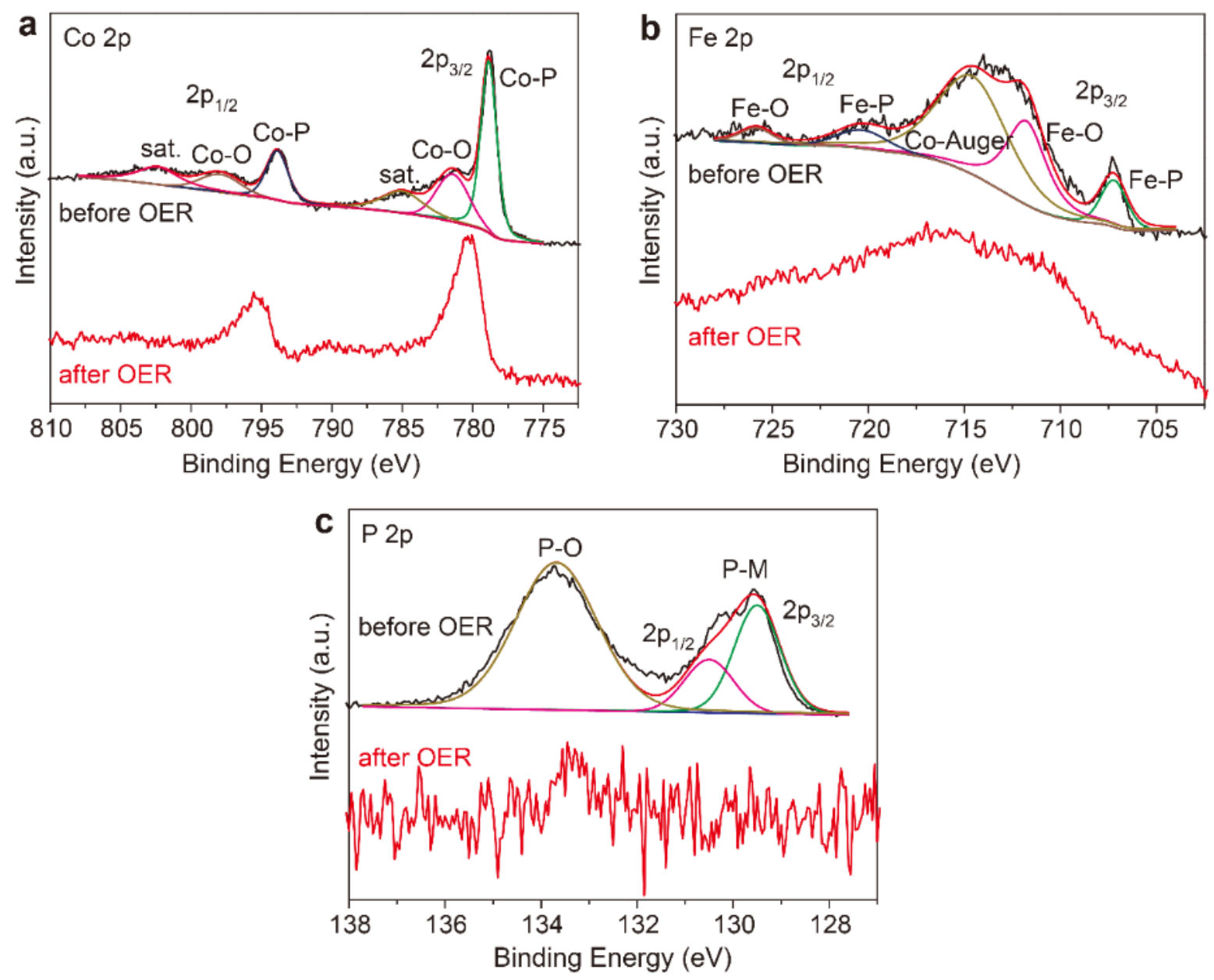

Figure S29. (a) Co $2 p$, (b) Fe $2 p$, and (c) P $2 p$ XPS spectra of the $\mathrm{rGO} / \mathrm{CoFe}-\mathrm{P}$ nanocomposite before and after OER stability test. 
Table S1. Comparison of the OER catalytic performance of a series of $\mathrm{rGO} / \mathrm{M}^{\mathrm{a}} \mathrm{M}^{\mathrm{b}}-\mathrm{P}$ (or $\mathrm{rGO} / \mathrm{M}^{\mathrm{a}} \mathrm{M}^{\mathrm{b}} \mathrm{M}^{\mathrm{c}}-\mathrm{P}$ ) nanocomposites in this work to other recently reported highperformance OER electrocatalysts in alkaline solution.

\begin{tabular}{|c|c|c|c|c|c|}
\hline Catalyst & $\begin{array}{l}\text { Mass loading } \\
\qquad\left(\mathrm{mg} \mathrm{cm} \mathbf{c m}^{-2}\right)\end{array}$ & Electrolyte & $\begin{array}{c}\eta @ 10 \mathrm{~mA} \mathrm{~cm} \mathbf{c}^{-2} \\
(\mathrm{mV})\end{array}$ & $\begin{array}{l}\text { Tafel slope } \\
\left(\mathrm{mV} \text { dec }^{-1}\right)\end{array}$ & Ref. \\
\hline rGO/CoFe-P & 0.1 & 1.0 М КОН & 270 & 38.3 & $\begin{array}{l}\text { this } \\
\text { work }\end{array}$ \\
\hline rGO/NiFe-P & 0.1 & 1.0 M KOH & 255 & 42.4 & $\begin{array}{l}\text { this } \\
\text { work }\end{array}$ \\
\hline rGO/CoNi-P & 0.1 & $1.0 \mathrm{M} \mathrm{KOH}$ & 314 & 60.0 & $\begin{array}{l}\text { this } \\
\text { work }\end{array}$ \\
\hline rGO/CoNiFe-P & 0.1 & $1.0 \mathrm{M} \mathrm{KOH}$ & 261 & 48.0 & $\begin{array}{l}\text { this } \\
\text { work }\end{array}$ \\
\hline $\begin{array}{l}\text { FeP nanorods/ } \\
\text { carbon paper }\end{array}$ & 0.7 & $1.0 \mathrm{M} \mathrm{KOH}$ & 350 & 63.6 & 1 \\
\hline FeP nanotubes & 1.6 & $1.0 \mathrm{M} \mathrm{KOH}$ & 288 & 43 & 2 \\
\hline CoP nanorods/C & 0.71 & $1.0 \mathrm{M} \mathrm{KOH}$ & 320 & 71 & 3 \\
\hline Co-P film & N.A. & $1.0 \mathrm{M} \mathrm{KOH}$ & 345 & 47 & 4 \\
\hline $\begin{array}{l}\text { Co-P/N-doped } \\
\text { Carbon }\end{array}$ & 0.283 & $1.0 \mathrm{M} \mathrm{KOH}$ & 354 & 52 & 5 \\
\hline $\mathrm{CoP} / \mathrm{rGO}$ & 0.28 & $1.0 \mathrm{M} \mathrm{KOH}$ & 340 & 66 & 6 \\
\hline CoP/NCNHP & N.A. & $1 \mathrm{M} \mathrm{KOH}$ & 310 & 70 & 7 \\
\hline Ni-P film & N.A. & $1.0 \mathrm{M} \mathrm{KOH}$ & 344 & 49 & 8 \\
\hline Ni-P nanoplates & 0.2 & $1.0 \mathrm{M} \mathrm{KOH}$ & 300 & 64 & 9 \\
\hline $\mathrm{Ni}_{2} \mathrm{P}$ nanoparticles & 0.14 & $1.0 \mathrm{M} \mathrm{KOH}$ & 290 & 59 & 10 \\
\hline $\mathrm{Ni}_{2} \mathrm{P}$ nanowires & 0.14 & $1.0 \mathrm{M} \mathrm{KOH}$ & 330 & 47 & 10 \\
\hline $\begin{array}{c}\text { multishelled } \mathrm{Ni}_{2} \mathrm{P} \\
\text { hollow microspheres }\end{array}$ & 0.283 & $1.0 \mathrm{M} \mathrm{KOH}$ & 270 & 40.4 & 11 \\
\hline $\mathrm{rGO} / \mathrm{Ni}_{2} \mathrm{P}$ & 0.1 & $1 \mathrm{M} \mathrm{KOH}$ & 283 & 43.6 & 12 \\
\hline $\mathrm{NiFeP}_{\mathrm{x}} @ \mathrm{NPS}-\mathrm{C}$ & 0.2 & $1.0 \mathrm{M} \mathrm{KOH}$ & 265 & 43.0 & 13 \\
\hline $\begin{array}{l}\mathrm{Ni}_{0.6} \mathrm{Co}_{1.4} \mathrm{P} \\
\text { nanocages }\end{array}$ & 0.35 & $1 \mathrm{M} \mathrm{KOH}$ & 300 & 80 & 14 \\
\hline CoMnP NPs & 0.28 & $1.0 \mathrm{M} \mathrm{KOH}$ & 330 & 61 & 15 \\
\hline $\mathrm{Fe}_{2-x} \mathrm{Mn}_{\mathrm{x}} \mathrm{P}$ nanorods & 0.284 & $1.0 \mathrm{M} \mathrm{KOH}$ & 440 & 39.0 & 16 \\
\hline $\begin{array}{c}\text { Nanoporous } \\
\left(\mathrm{Co}_{0.52} \mathrm{Fe}_{0.48}\right)_{2} \mathrm{P}\end{array}$ & N.A. & $1.0 \mathrm{M} \mathrm{KOH}$ & 270 & 30 & 17 \\
\hline $\begin{array}{l}\text { sea-urchin-like } \\
\left(\mathrm{Co}_{0.54} \mathrm{Fe}_{0.46}\right) \mathrm{P}_{2}\end{array}$ & 0.2 & $0.1 \mathrm{M} \mathrm{KOH}$ & 370 & N.A. & 18 \\
\hline $\mathrm{NiCoP}$ & 1.6 & $1.0 \mathrm{M} \mathrm{KOH}$ & 280 & 87 & 19 \\
\hline $\mathrm{rGO} / \mathrm{NiCoP}$ & 0.15 & $1.0 \mathrm{M} \mathrm{KOH}$ & 270 & 65.7 & 20 \\
\hline
\end{tabular}




\begin{tabular}{|c|c|c|c|c|c|}
\hline $\mathrm{Ni}_{2} \mathrm{P}-\mathrm{CoP}$ & 0.2 & $0.1 \mathrm{M} \mathrm{KOH}$ & 320 & 69 & 21 \\
\hline $\begin{array}{l}\mathrm{Ag}-\mathrm{CoSe}_{2} \\
\text { nanobelts }\end{array}$ & 0.2 & $0.1 \mathrm{M} \mathrm{KOH}$ & 320 & 56 & 22 \\
\hline CoSn-hydroxide & 0.66 & $1.0 \mathrm{M} \mathrm{KOH}$ & 274 & N.A. & 23 \\
\hline $\begin{array}{c}\text { CoAl- } \\
\text { LDH/graphene } \\
\text { network }\end{array}$ & 0.05 & $1.0 \mathrm{M} \mathrm{KOH}$ & 301 & 43 & 24 \\
\hline $\mathrm{NiFeO}_{\mathrm{x}}$ film & N.A. & $1.0 \mathrm{M} \mathrm{NaOH}$ & $>350$ & N.A. & 25 \\
\hline $\mathrm{Ni}_{0.9} \mathrm{Fe}_{0.1} / \mathrm{N}-\mathrm{C}$ & 0.2 & $1.0 \mathrm{M} \mathrm{KOH}$ & 330 & 45 & 26 \\
\hline $\begin{array}{c}\mathrm{Co}_{3} \mathrm{O}_{4} / \mathrm{C} \text { nanowire } \\
\text { arrays }\end{array}$ & 0.2 & $1.0 \mathrm{M} \mathrm{KOH}$ & 220 & 61 & 27 \\
\hline $\begin{array}{c}\mathrm{Zn}_{\mathrm{X}} \mathrm{Co}_{3-\mathrm{x}} \mathrm{O}_{4} \\
\text { nanowire arrays }\end{array}$ & 1.0 & $1.0 \mathrm{M} \mathrm{KOH}$ & 320 & 51 & 28 \\
\hline $\begin{array}{c}\mathrm{Ni}_{\mathrm{x}} \mathrm{Co}_{3-\mathrm{x}} \mathrm{O}_{4} \\
\text { nanowire arrays }\end{array}$ & 2.3 to 2.7 & $1.0 \mathrm{M} \mathrm{NaOH}$ & 370 & $59-64$ & 29 \\
\hline $\begin{array}{c}\text { Ni-Co oxide } \\
\text { nanosheets }\end{array}$ & N.A. & $1.0 \mathrm{M} \mathrm{NaOH}$ & 340 & 51 & 30 \\
\hline $\begin{array}{c}\text { Amorphous } \\
\mathrm{NiCo}_{2.7}(\mathrm{OH})_{\mathrm{x}} \\
\text { nanocages }\end{array}$ & 0.2 & $1.0 \mathrm{M} \mathrm{KOH}$ & 350 & 65 & 31 \\
\hline $\begin{array}{c}\text { Amorphous Ni-Co } \\
\text { binary oxide }\end{array}$ & N.A. & $1.0 \mathrm{M} \mathrm{NaOH}$ & 325 & 39 & 32 \\
\hline $\begin{array}{l}\mathrm{Ni}-\mathrm{Co} \text { mixed oxide } \\
\text { cages }\end{array}$ & N.A. & $1.0 \mathrm{M} \mathrm{KOH}$ & 380 & 50 & 33 \\
\hline $\begin{array}{l}\text { NiCo LDH } \\
\text { nanosheets }\end{array}$ & 0.17 & $1.0 \mathrm{M} \mathrm{KOH}$ & 367 & 40 & 34 \\
\hline $\begin{array}{c}\text { Ultrathin } \mathrm{NiCo}_{2} \mathrm{O}_{4} \\
\text { nanosheets }\end{array}$ & 0.285 & $1.0 \mathrm{M} \mathrm{KOH}$ & 320 & 30 & 35 \\
\hline $\begin{array}{c}\text { N-doped graphitic } \\
\text { carbon }\end{array}$ & 0.2 & $0.1 \mathrm{M} \mathrm{KOH}$ & 380 & $75-80$ & 36 \\
\hline $\begin{array}{c}\text { P-doped graphitic } \\
\mathrm{C}_{3} \mathrm{~N}_{4}\end{array}$ & 0.2 & $0.1 \mathrm{M} \mathrm{KOH}$ & 400 & 61.6 & 37 \\
\hline $\begin{array}{c}\text { Graphitic } \mathrm{C}_{3} \mathrm{~N}_{4} \\
\text { nanosheets/carbon } \\
\text { nanotubes }\end{array}$ & 0.2 & $0.1 \mathrm{M} \mathrm{KOH}$ & 370 & 83 & 38 \\
\hline $\mathrm{IrO}_{\mathrm{x}}$ & N.A. & $1.0 \mathrm{M} \mathrm{NaOH}$ & $\begin{array}{c}320 \pm 40(\text { not } \\
\text { stable })\end{array}$ & N.A. & 25 \\
\hline $\mathrm{IrO}_{\mathrm{x}}$ & N.A. & $1.0 \mathrm{M} \mathrm{KOH}$ & $427 \pm 5$ & $49 \pm 1$ & 39 \\
\hline $\mathrm{IrO}_{2}$ & 0.21 & $1.0 \mathrm{M} \mathrm{KOH}$ & 338 & 47 & 40 \\
\hline
\end{tabular}




\section{References:}

(1) Xiong, D.; Wang, X.; Li, W.; Liu, L. Chem. Commun. 2016, 52, 8711.

(2) Yan, Y.; Xia, B. Y.; Ge, X.; Liu, Z.; Fisher, A.; Wang, X. Chemistry - A European Journal 2015, 21, 18062.

(3) Chang, J.; Xiao, Y.; Xiao, M.; Ge, J.; Liu, C.; Xing, W. ACS Catal. 2015, 5, 6874.

(4) Jiang, N.; You, B.; Sheng, M.; Sun, Y. Angew. Chem. Int. Ed. 2015, 54, 6251.

(5) You, B.; Jiang, N.; Sheng, M.; Gul, S.; Yano, J.; Sun, Y. Chem. Mater. 2015, $27,7636$.

(6) Jiao, L.; Zhou, Y.-X.; Jiang, H.-L. Chem. Sci. 2016, 7, 1690.

(7) Pan, Y.; Sun, K.; Liu, S.; Cao, X.; Wu, K.; Cheong, W.-C.; Chen, Z.; Wang, Y.; Li, Y.; Liu, Y.; Wang, D.; Peng, Q.; Chen, C.; Li, Y. J. Am. Chem. Soc. 2018, 140, 2610.

(8) Jiang, N.; You, B.; Sheng, M.; Sun, Y. ChemCatChem 2016, 8, 106.

(9) Yu, X.-Y.; Feng, Y.; Guan, B.; Lou, X. W.; Paik, U. Energy Environ. Sci. 2016, 9, 1246.

(10)Stern, L.-A.; Feng, L.; Song, F.; Hu, X. Energy Environ. Sci. 2015, 8, 2347.

(11) Sun, H.; Xu, X.; Yan, Z.; Chen, X.; Cheng, F.; Weiss, P. S.; Chen, J. Chem. Mater. 2017, 29, 8539.

(12)Li, P.; Chen, R.; Tian, S.; Xiong, Y. ACS Sustainable Chemistry \& Engineering 2019, 7, 9566 .

(13)Li, P.; Zeng, H. C. Chem. Commun. 2017, 53, 6025.

(14)Bocheng, Q.; Lejuan, C.; Yang, W.; Ziyuan, L.; Yunpeng, Z.; Mengye, W.; Yang, C. Adv. Funct. Mater. 2018, 28, 1706008.

(15)Li, D.; Baydoun, H.; Verani, C. N.; Brock, S. L. J. Am. Chem. Soc. 2016, 138, 4006.

(16)Li, D.; Baydoun, H.; Kulikowski, B.; Brock, S. L. Chem. Mater. 2017, 29, 3048.

(17)Tan, Y.; Wang, H.; Liu, P.; Shen, Y.; Cheng, C.; Hirata, A.; Fujita, T.; Tang, Z.; Chen, M. Energy Environ. Sci. 2016, 9, 2257.

(18)Mendoza-Garcia, A.; Zhu, H.; Yu, Y.; Li, Q.; Zhou, L.; Su, D.; Kramer, M. J.; Sun, S. Angew. Chem. Int. Ed. 2015, 54, 9642. 
(19)Liang, H.; Gandi, A. N.; Anjum, D. H.; Wang, X.; Schwingenschlögl, U.; Alshareef, H. N. Nano Lett. 2016, 16, 7718.

(20)Li, J.; Yan, M.; Zhou, X.; Huang, Z.-Q.; Xia, Z.; Chang, C.-R.; Ma, Y.; Qu, Y. Adv. Funct. Mater. 2016, 26, 6785.

(21)Liang, X.; Zheng, B.; Chen, L.; Zhang, J.; Zhuang, Z.; Chen, B. ACS Appl. Mater. Interfaces 2017, 9, 23222.

(22)Zhao, X.; Zhang, H.; Yan, Y.; Cao, J.; Li, X.; Zhou, S.; Peng, Z.; Zeng, J. Angew. Chem. Int. Ed. 2017, 56, 328.

(23)Song, F.; Schenk, K.; Hu, X. Energy Environ. Sci. 2016, 9, 473.

(24)Ping, J.; Wang, Y.; Lu, Q.; Chen, B.; Chen, J.; Huang, Y.; Ma, Q.; Tan, C.; Yang, J.; Cao, X.; Wang, Z.; Wu, J.; Ying, Y.; Zhang, H. Adv. Mater. 2016, 28, 7640.

(25)McCrory, C. C. L.; Jung, S.; Peters, J. C.; Jaramillo, T. F. J. Am. Chem. Soc. 2013, $135,16977$.

(26)Zhang, X.; Xu, H.; Li, X.; Li, Y.; Yang, T.; Liang, Y. ACS Catal. 2016, 6, 580.

(27)Ma, T. Y.; Dai, S.; Jaroniec, M.; Qiao, S. Z. J. Am. Chem. Soc. 2014, 136, 13925.

(28)Liu, X.; Chang, Z.; Luo, L.; Xu, T.; Lei, X.; Liu, J.; Sun, X. Chem. Mater. 2014, $26,1889$.

(29)Li, Y.; Hasin, P.; Wu, Y. Adv. Mater. 2010, 22, 1926.

(30)Wang, H.-Y.; Hsu, Y.-Y.; Chen, R.; Chan, T.-S.; Chen, H. M.; Liu, B. Adv. Energy Mater. 2015, 5, 1500091.

(31)Nai, J.; Yin, H.; You, T.; Zheng, L.; Zhang, J.; Wang, P.; Jin, Z.; Tian, Y.; Liu, J.; Tang, Z.; Guo, L. Adv. Energy Mater. 2015, 5.

(32) Yang, Y.; Fei, H.; Ruan, G.; Xiang, C.; Tour, J. M. ACS Nano 2014, 8, 9518.

(33)Han, L.; Yu, X. Y.; Lou, X. W. Adv. Mater. 2016, 28, 4601.

(34)Liang, H.; Meng, F.; Cabán-Acevedo, M.; Li, L.; Forticaux, A.; Xiu, L.; Wang, Z.; Jin, S. Nano Lett. 2015, 15, 1421.

(35)Bao, J.; Zhang, X.; Fan, B.; Zhang, J.; Zhou, M.; Yang, W.; Hu, X.; Wang, H.; Pan, B.; Xie, Y. Angew. Chem. Int. Ed. 2015, 54, 7399.

(36)Zhao, Y.; Nakamura, R.; Kamiya, K.; Nakanishi, S.; Hashimoto, K. Nat 
Commun 2013, 4, 2390.

(37)Ma, T. Y.; Ran, J.; Dai, S.; Jaroniec, M.; Qiao, S. Z. Angew. Chem. Int. Ed. 2015, 54, 4646.

(38)Ma, T. Y.; Dai, S.; Jaroniec, M.; Qiao, S. Z. Angew. Chem. Int. Ed. 2014, 53, 7281.

(39) Trotochaud, L.; Ranney, J. K.; Williams, K. N.; Boettcher, S. W. J. Am. Chem. Soc. 2012, 134, 17253.

(40)Song, F.; Hu, X. Nat Commun 2014, 5, 4477. 